\title{
HARMONICALLY INDUCED REPRESENTATIONS ON NILPOTENT LIE GROUPS AND AUTOMORPHIC FORMS ON NILMANIFOLDS
}

BY

\author{
RICHARD C. PENNEY
}

\begin{abstract}
It is shown that the irreducible "discrete series" representations of certain nilpotent Lie groups may be realized in square integrable $\bar{\partial}$ cohomology spaces. This theory is applied to obtain a concept of automorphic forms on nilmanifolds which generalizes the niltheta functions of Cartier and AuslanderTolimieri. We also use the automorphic cohomology to solve certain holomorphic difference equations on $\mathbf{C}^{\mathbf{n}}$.
\end{abstract}

I. Introduction. Let $\Re$ be a nilpotent Lie group and let $\lambda \in \Re^{*}$. Let $\Re_{c}$ be the complexification of $\Re$ and let $\lambda$ denote also the extension of $\lambda$ to $\Re_{c}$. A subalgebra $\mathscr{P}$ of $\mathscr{T}_{c}$ is subordinate to $\lambda$ if $[\mathscr{P}, \mathscr{P}] \subset \operatorname{ker} \lambda . \mathscr{P}$ is maximal subordinate if $\mathcal{P}$ is of maximal dimension among the subordinate subalgebras of $\Re . \mathcal{P}$ is said to be totally complex if $\mathscr{P}+\overline{\mathcal{P}}=\Re_{c}$ and $\mathscr{P} \cap \overline{\mathscr{P}}$ is one dimensional. In this paper we shall consider a fixed triple $(\mathcal{R}, \lambda, \mathscr{P})$ consisting of a nilpotent Lie algebra $\Re$, a functional $\lambda$ and a totally complex, maximal subordinate subalgebra $\mathscr{P}$. We shall adopt the convention that Lie algebras will be denoted by upper case script letters and the corresponding connected, simply connected Lie group will be denoted by the corresponding upper case Latin letter.

Let $\mathcal{Z}$ be the center of $\mathcal{~}$. We define a character $\chi$ of $Z$ by setting $\chi(z)=$ $\exp i \lambda\left(\log _{N} z\right)$. Let $N^{\prime}=Z \backslash N$ and let $\pi: N \rightarrow N^{\prime}$ be the natural projection. Following Satake [21] we define a line bundle $E$ over $N^{\prime}$ as follows. Let $E_{0}=N \times$ C. $Z$ acts on $E_{0}$ according to $(n, c) z=(n z, \chi(z) c)$. Let $E=E_{0} / Z$. Let $p: E \rightarrow N^{\prime}$ be defined by $p([(n, c)])=\pi(n)$ where "[ ]" denotes equivalent class. Then $(E, p$, $N^{\prime}$ ) is a complex vector bundle with fiber isomorphic with C. $N$ acts on $E_{0}$ on both the left and the right according to $g(n, \alpha)=(g n, \alpha),(n, \alpha) g=(n g, \alpha)$ and these actions factor down to actions on $E$. $E$ carries an $N$-invariant Hermitian structure defined by $\left([(n, c)],\left[\left(n^{\prime}, c^{\prime}\right)\right]\right)_{E}=c \bar{c}^{\prime}$.

Let $\mathcal{U} \subset N^{\prime}$ be open. We will let $\Gamma_{\infty}(\mathcal{U}, E)$ denote the $C^{\infty}$ sections of $E$ over U. $\Gamma_{\infty}(\mathcal{U}, E)$ may be canonically identified with the space of $C^{\infty}$ mappings $f$ of $\pi^{-1}(\mathscr{Q}) \subset N$ into $C$ which satisfy $f(x z)=\chi(z) f(x)$ for all $x \in N$ and $z \in Z$. One identifies $f \in \Gamma_{\infty}(\mathscr{Q}, E)$ with the function $\tilde{f}$ defined by $\tilde{f}(n)=\alpha$ if $f(\pi(n))=$ $[(n, \alpha)]$. We shall usually make this identification without comment.

Let $X \in \mathfrak{R}$. We define a mapping $\nabla_{X}: \Gamma_{\infty}(\mathcal{Q}, E) \rightarrow \Gamma_{\infty}(\mathcal{Q}, E)$ by $\nabla_{X} f(n)=X$ . $f(n)-i \lambda(X) f(n)$ where " $X$ " represents the action of $X$ on $C^{\infty}\left(\pi^{-1}(\mathcal{Q})\right)$ as a left

Received by the editors January 11, 1979.

AMS (MOS) subject classifications (1970). Primary 22E25, 22E40, 22E45, 43A85. 
invariant differential operator. It is easily verified that $\nabla_{X}$ depends only on the coset of $X \bmod \Psi$ and $\nabla$ may be extended to a connection of $E$ over $N^{\prime}$.

Now, as pointed out by Satake, $N^{\prime}$ has a natural complex structure. In fact $\Re+\overline{\mathscr{P}}=\mathscr{K}_{c}$ and $\mathscr{T} \cap \overline{\mathcal{P}}=\mathscr{Z}$ so $N^{\prime}=N_{c} / \bar{P}$ as a homogeneous space. The isomorphism defines the complex structure. The canonical splitting of the complexified tangent bundle of $N^{\prime}$ associated with this structure is provided by $\Re_{c}^{\prime}=\mathscr{P} / \mathscr{Z}_{c} \oplus \overline{\mathcal{P}} / \mathscr{Z}_{c}$.

Let $\Gamma^{p}(E)$ denote the set of all complex $p$-linear alternating maps of $\left(\overline{\mathcal{P}} / \Psi_{c}\right)^{p}$ into $\Gamma_{\infty}(E)$. Due to the triviality of the tangent bundle of $N^{\prime}, \Gamma^{p}(E)$ is canonically isomorphic with the space of $E$ valued forms of type $(0, p)$ on $N^{\prime}$. We define a mapping $\bar{\partial}: \Gamma^{p}(E) \rightarrow \Gamma^{p+1}(E)$ by

$$
\begin{aligned}
\bar{\partial} f\left(X_{1}, \ldots, X_{p+1}\right)= & \sum_{i=1}^{p+1}(-1)^{i} \nabla_{X_{i}} f\left(X_{1}, \ldots, X_{i}, \ldots, X_{p+1}\right) \\
& +\sum_{i<j}(-1)^{i+j} f\left(\left[X_{i}, X_{j}\right], X_{1}, \ldots, X_{i}, \ldots, X_{j}, \ldots, X_{p+1}\right)
\end{aligned}
$$

for $X_{i} \in \overline{\mathcal{P}} / \mathscr{Z}_{c}$

Now, let $C^{0}(E)$ denote the largest subspace of $\Gamma_{\infty}(E)$ such that

(1) $C^{0}(E)$ is invariant under $\nabla_{X}$ for all $X \in \overline{\mathcal{P}} / \mathscr{Z}_{c}$.

(2) For all $f \in C^{0}(E)$, the function

$$
x \rightarrow(f(x), f(x))_{E}=\|f(x)\|_{E}^{2} \text { is in } L^{1}\left(N^{\prime}\right) .
$$

Let $C^{p}(E)$ be the subspace of $\Gamma^{p}(E)$ of forms with coefficients in $C^{0}(E)$. Then $\bar{\partial}$ : $C^{p}(E) \rightarrow C^{p+1}(E)$.

Now suppose $N_{c}^{\prime}$ is equipped with a positive definite Hermitian structure. We then get a Hermitian structure on $\Lambda^{p}\left(\left(\overline{\mathcal{P}} / \mathscr{Z}_{c}\right)^{*}\right)$. This Hermitian structure defines (by integration over $N^{\prime}$ ) a scalar product $(,)_{p}$ on $\Gamma^{p}(E) \times \Gamma^{p}(E)$ (see [21] for more details). The elements of $C^{p}(E)$ have finite $(,)_{p}$ norm and it follows by standard computations that $\bar{\partial}: C^{p}(E) \rightarrow C^{p+1}(E)$ has a formal adjoint $\delta: C^{p+1}(E) \rightarrow C^{p}(E)$.

Let $L_{2}^{p}(E)$ be the completion of $C^{p}(E)$ in \|\|$_{p}$ and let $\bar{\partial}_{c}$ and $\delta_{c}$ denote, respectively, the adjoints of $\delta$ and $\bar{\partial}$ in \|\|$_{p}$. Let $\square$ be the partially defined mapping of $L_{2}^{p}(E)$ into itself defined by $\square=\partial_{c} \delta_{c}+\delta_{c} \partial_{c}$. Let $\mathcal{H}^{p}(E)=\operatorname{ker} \square \cdot \mathcal{H}^{p}(E)$ is referred to as the space of harmonic forms of degree $p$. $\mathcal{H}^{p}(E)$ is a closed subspace of $L_{2}^{p}(E)$. The left action of $N$ on $E$ gives rise to a unitary representation of $N$ in $L_{2}^{p}(E)$ and this representation clearly leaves $\mathcal{F C}^{p}(E)$ invariant. Let $T$ denote the restriction of this representation to $\mathcal{H}^{p}(E)$. As Satake points out, it is a fundamental problem of representation theory to decide when $\mathcal{F C}^{p}(E)$ is nontrivial and when $T$ is irreducible. A conjecture of Langlands states that there should exist a unique $p$, depending on $\mathcal{P}$ and $\lambda$, such that $\mathcal{H}^{p}(E)$ is nonzero. Furthermore, for this unique $p, T$ should also be irreducible.

This conjecture has been verified in the semisimple case by Schmid [22]. In the nilpotent case the conjecture has been studied by Satake [21] and Camora [3] in the Heisenberg case and by Moscovici [15] and Moscovici-Verona [14] in general. Moscovici-Verona show that the conjecture is true under the conditions that $\lambda$ is sufficiently far from 0 and $\Re$ has a rational structure which relates nicely to $\mathscr{P}$ 
and $\lambda$. Moscovici has also shown the vanishing part of the theorem for $\lambda$ sufficiently far from 0 without any rationality assumptions. It is our goal in this work to prove the conjecture without any special assumptions on $\mathscr{P}$ or $\lambda$. At the moment we are only able to handle the case that $\mathscr{P}$ is abelian, although we feel that our techniques do present a viable approach to the general case as well. ${ }^{1}$

The main technique of proof is to relate the analytically defined spaces $\mathcal{F}^{p}(E)$ to certain Lie algebra cohomology spaces defined from unitary representations. We then use the Hochschild-Serre spectral theorem to compute the Lie algebra cohomology. Our approach resembles the approach used by Schmid in the semisimple case. However, the passage from analysis to algebra is complicated by the facts that we do not have any central elliptic elements in the enveloping algebra and we do not have the space of $K$-finite vectors at our disposal. What we use instead is the space of $C^{\infty}$ vectors and the corresponding $C^{\infty}$ regularity theory of elliptic operators as developed by Nelson and Goodman ([16], [6]). As an application, we follow the familiar route from harmonic induction to automorphic forms on compact homogeneous spaces. Specifically, suppose there is a discrete, cocompact subgroup $\Gamma \subset N$ such that $\Gamma \cap Z \subset \operatorname{ker} \chi$. (These assumptions are equivalent to the assumption that $\Re$ and $\lambda$ are rational.) Let $\Delta=\Gamma Z$ and let $\mu$ be the character of $\Delta$ defined by $\mu(\gamma z)=\chi(z)$.

Let $M=\Delta \backslash N$. We use the pair $(\mu, \Delta)$ to define a vector bundle $F$ over $M$ just as $E$ was defined from $(Z, \chi)$. We define a connection $\nabla$ on $F$ just as we did for $E$. The spaces $C^{P}(F)$ are then defined just as before. (Instead of Haar measure on $N^{\prime}=N / Z$ we use the canonical $N$-invariant probability measure on $\Delta \backslash N$. Note however that $C^{0}(F)=\Gamma_{\infty}(X, F)$, as continuous functions are automatically $L^{2}$.) We define $\mathcal{H}^{p}(F)$ as before. Due to compactness, $\mathcal{F}^{p}(F)$ is finite dimensional and $H^{p}(F)$ (the DeRahm cohomology of $F$ ) and $\mathcal{F}^{p}(F)$ are isomorphic. We show that if $\mathscr{P}$ is abelian, $\mathcal{F}^{p}(F)$ is nonzero for precisely one $p$ and for this $p$ the dimension of $\mathcal{H}^{p}(F)$ is the multiplicity of the representation corresponding to $\lambda$ under the Kirillov correspondence in the quasi-regular representation of $N$ in $L^{2}(\Gamma \backslash N)$. The nonzero elements of $H^{p}(F)$ are by definition the automorphic cohomology classes of $M$ and the elements of $\mathcal{H}^{P}(E)$ are the harmonic automorphic forms. It is interesting that the theta functions of Auslander-Tolimieri are special cases of the harmonic automorphic forms.

The spaces $H^{p}(F)$ are isomorphic with certain holomorphic $\Gamma$-cohomology spaces. This isomorphism gives rise to what seems to us to be a fascinating collection of theorems concerning certain "holomorphic difference equations". For example, the statement that $H^{1}(F)=0$ for the Heisenberg group implies the following

THEOREM 1. Consider the following difference equation on $\mathbf{C}$ where $f_{1}$ and $f_{2}$ are entire functions:

$$
f_{1}(z+i)-f_{1}(z)=e^{-2 \pi(z+1 / 2)} f_{2}(z+1)-f_{2}(z) .
$$

\footnotetext{
${ }^{1}$ Note added in proof. The general case has recently been done independently by the author and $\mathrm{J}$. Rosenberg. The technique is somewhat different than the one presented here.
} 
Then there is an entire function $g$ such that

$$
f_{1}(z)=e^{-2 \pi(z+1 / 2)} g(z+1)-g(z), \quad f_{2}(z)=g(z+i)-g(z) .
$$

Furthermore, for $f_{1}$ and $f_{2}$ given, the set of $g$ which so represents $f$ is one dimensional.

As mentioned above, this type theorem is a consequence of an isomorphism between $H^{p}(F)$ and certain group-theoretic cohomology groups defined from $\Gamma$. Presumably such isomorphisms are well known in the semisimple case, although they are new to us.

II. Generalities. In this section we forge the connection between the analysis of $\S I$ and the algebra of spectral sequences. First we describe a construction which generalize the constructions described in \$I. (See Schmid [22].)

Let $U$ be a unitary representation of $N$ in a Hilbert space $\mathcal{H}=\mathcal{H}(U)$. Let $C^{\infty}(U)$ be the space of $C^{\infty}$ vectors of $U$ given its usual Fréchet space topology [17]. For brevity we shall set $C=C^{\infty}(U)$. We shall also denote the representation of $\Re_{c}$ in $C$ defined by $U$ by juxtaposition so $X v=\partial U(X) v$ for $X \in \Re_{c}$ and $v \in C$. Let $\Re_{0}$ be any subalgebra of $\Re_{c}$. We let $C^{p}\left(\Re_{0}, C\right)$ be the set of complex $p$-linear, alternating mappings of $\left(\Re_{0}\right)^{p}$ into $C$. We define the differential $\bar{\partial}$ : $C^{p}\left(\Re_{0}, C\right) \rightarrow C^{p+1}\left(\Re_{0}, C\right)$ by

$$
\begin{aligned}
\bar{\partial} f\left(X_{1}, \ldots, X_{p+1}\right)= & \sum_{i=1}^{n}(-1)^{i+1} X_{i} f\left(X_{1}, \ldots, X_{i}, \ldots, X_{p+1}\right) \\
& +\sum_{i<j}(-1)^{i+j} f\left(\left[X_{i}, X_{j}\right], X_{1}, \ldots, X_{i}, \ldots, X_{j}, \ldots, X_{p+1}\right) .
\end{aligned}
$$

$\bar{\partial}$ is merely the Hochschild-Serre coboundary operator for the $\Re_{0}$-module $C$ [8].

Now, suppose $($,$) is a positive definite Hermitian form on \Re_{c}$. Then $($,$) gives$ rise to a scalar product on $C^{p}\left(\Re_{0}, C\right)$ as follows. Let $\left(E_{1}, \ldots, E_{n}\right)=\mathcal{E}$ be an ordered orthonormal basis of $\Re_{0}$. Let $B(p)$ be the set of multi-indices $\alpha$ of length $p$ with $0 \leqslant \alpha(0)<\alpha(1)<\cdots<\alpha(p) \leqslant n$. For $\alpha \in B(p)$ let $E_{\alpha}=\left(E_{\alpha(1)}\right.$, $\left.E_{\alpha(2)}, \ldots, E_{\alpha(p)}\right) \in\left(\mathscr{T}_{0}\right)^{p}$. Let $(f, g)_{p}=\Sigma_{\alpha \in B(p)}\left(f\left(E_{\alpha}\right), g\left(E_{\alpha}\right)\right)$ for $f, g \in$ $C^{p}\left(\mathscr{T}_{0}, C\right)$. The inner product in the sum is the $\mathscr{H}(U)$ inner product. It can be seen that $(,)_{p}$ does not depend on the orthonormal basis $E$.

LEMMA 2. The operator $\bar{\partial}: C^{p}\left(\Re_{0}, C\right) \rightarrow C^{p+1}\left(\Re_{0}, C\right)$ has a formal adjoint $\delta$ : $C^{p+1}\left(\Re_{0}, C\right) \rightarrow C^{p}\left(\Re_{0}, C\right)$ relative to $(,)_{p}$.

Proof. This is proved in [22]. However, for later purposes, we include a proof. Note that as inner product spaces,

$$
C^{p}\left(\Re_{0}, C\right) \approx C \otimes \Lambda^{p}\left(\Re_{0}^{*}\right)=C \otimes C^{p}\left(\Re_{0}, 1\right)
$$

where 1 represents the trivial action of $\Re_{0}$ on $C$. The $\bar{\partial}$ operator is a sum of the operators $I \otimes \bar{\partial}_{1}$ and $D$ where $\bar{\partial}_{1}$ is the coboundary operator of $C^{p}\left(\Re_{0}, 1\right)$ and $D$ is defined by

$$
D f\left(X_{1}, \ldots, X_{p+1}\right)=\sum(-1)^{i+1} X_{i} f\left(X_{1}, \ldots, X_{i}, \ldots, X_{p+1}\right) .
$$


$I \otimes \bar{\partial}_{1}$ has an adjoint by the finite dimensionality of $C^{p}\left(\Re_{0}, 1\right)$ and the adjoint $D^{*}$ of $D$ is easily computed to be

$$
D^{*} f\left(X_{1}, \ldots, X_{p}\right)=-\sum \bar{E}_{i} f\left(E_{i}, X_{1}, \ldots, X_{p}\right)
$$

where $\bar{E}_{i}$ is the conjugate of $E_{i}$ in $\Re_{c}$. (Note that $E_{i}^{*}=-\bar{E}_{i}$ on $C$.) Q.E.D.

Now let $C^{p}\left(\mathscr{T}_{0}, \mathcal{H}\right)$ be the space of $\mathcal{H}$-valued, complex, alternating, $p$-linear maps on $\left(\mathscr{T}_{0}\right)^{p}$. We give $C^{p}\left(\mathscr{T}_{0}, \mathcal{K}\right)$, the scalar product $(,)_{p}$ defined analogously to $(,)_{p}$ on $C^{p}\left(\Re_{0}, C\right) . C^{p}\left(\mathscr{T}_{0}, \mathcal{H}_{C}\right)$ is then the completion of $C^{p}\left(\Re_{0}, C\right)$. We define $\bar{\partial}_{c}$ and $\delta_{c}$ to be the adjoints of $\delta$ and $\bar{\partial}$ respectively on $C^{p}\left(\mathscr{T}_{0}, \mathcal{K}\right)$ and we define to be the partially defined operator on $C^{p}\left(\Re_{0}, \mathcal{H}\right)$ given by $\square=\bar{\partial}_{c} \delta_{c}+\delta_{c} \bar{\partial}_{c}$. We let $\mathcal{F}^{p}\left(\mathscr{T}_{0}, U\right)=\operatorname{ker} \square . \mathcal{H}^{p}\left(\mathscr{T}_{0}, U\right)$ is a closed subspace of $C^{p}\left(\mathscr{T}_{0}, \mathcal{H}\right)$. In fact, it follows from standard reasoning that $\mathcal{H}^{p}\left(\Re_{0}, U\right)$ is the intersection of the kernels of the closed operators of $\delta_{c}$ and $\partial_{c}$.

We claimed that the above construction generalized the construction of $\mathcal{H}^{p}(F)$ and $\mathcal{H}^{p}(E)$. To see this let $U^{\chi}=\operatorname{ind}(Z, N, \chi)$ and $U^{\mu}=\operatorname{ind}(\Delta, N, \mu)$ where $\chi$ and $\mu$ are as in the introduction and "ind" indicates induced representation. We realize our induced representations as usual by right translation in spaces of functions which transform on the left according to the appropriate character of the appropriate subgroup and which satisfy the appropriate integrability condition (see [2]). Let $C^{x}$ and $C^{\mu}$ be the $C^{\infty}$ spaces of $U^{x}$ and $U^{\mu}$ respectively. It follows from results of Poulsen [19], that $C^{\chi}$ is precisely the space $C^{0}(E)$ defined in $\S I$ and $C^{\mu}$ is precisely $C^{0}(F)$. Let $\mathscr{P}_{0}=\overline{\mathscr{P}} \cap \operatorname{ker} \lambda$ in $\mathscr{T}_{c} \cdot \mathscr{P}_{0}$ is a subalgebra and $\overline{\mathscr{P}} / \mathscr{I}_{c} \approx \mathscr{P}_{0}$. It is obvious that

$$
\mathcal{H}^{P}(F) \approx \mathcal{H}^{p}\left(\mathscr{P}_{0}, C^{X}\right), \quad \mathcal{H}^{p}(E) \approx \mathcal{H}^{P}\left(\mathscr{P}_{0}, C^{\mu}\right) .
$$

The "algebraically" defined spaces we shall relate $\mathcal{F}^{p}\left(\Re_{0}, U\right)$ to are the cohomology spaces $H^{p}\left(\mathscr{T}_{0}, C\right)$ of the complex $\left(C^{p}\left(\mathscr{T}_{0}, C\right), \bar{\partial}\right)$ defined by

$$
H^{p}\left(\Re_{0}, C\right)=\operatorname{ker} \bar{\partial} / \operatorname{im} \bar{\partial} \text { in } C^{p}\left(\Re_{0}, C\right) .
$$

$C^{p}\left(\Re_{0}, C\right)$ has a natural Fréchet space topology defined from the $C^{\infty}$ topology of $C$ and the identification of $C^{p}\left(\Re_{0}, C\right)$ with $C \otimes \Lambda^{p}\left(\Re_{0}^{*}\right)$. We shall give $H^{p}\left(\Re_{0}, C\right)$ the quotient topology. We shall also consider the space

$$
\underline{H}^{p}\left(\Re_{0}, C\right)=\operatorname{ker} \bar{\partial} /[\operatorname{im} \bar{\partial}]_{\infty}
$$

in $C^{p}\left(\Re_{0}, C\right)$ where [ $]_{\infty}$ indicates closure in $C^{p}\left(\Re_{0}, C\right)$. The topology of $H^{p}\left(\Re_{0}, C\right)$ need not be Hausdorff although that of $\underline{H}^{p}\left(\Re_{0}, C\right)$ of course is. Our main result of this section is the following analogue of Theorem 3.1 of [22]. Note that our proof is valid for any Lie group which possesses a suitable subalgebra $\mathscr{P}_{0}$.

THEOREM 3. $H^{p}\left(\mathscr{P}_{0}, C\right)$ and $\mathcal{S C}^{p}\left(\mathscr{P}_{0}, U\right)$ are isomorphic. In fact ker $\bar{\partial}=$ $\mathcal{H}^{p}\left(\mathscr{P}_{0}, U\right) \oplus[\operatorname{Im} \bar{\partial}]_{\infty}$.

Proof. The main step is the following proposition (see Corollary 2.21 of [22]).

Proposition 4. $C^{p}\left(\mathscr{P}_{0}, C\right)=\bigcap_{n}$ domain $\square^{n}$. 
This proposition is a generalization of results of Goodman (cf. [6, Theorem]). To prove it we shall generalize Goodman's arguments. Specifically, let $\mathscr{K}$ be the Hilbert space $\Lambda^{p}\left(\mathscr{P}_{0}^{*}\right)$. Let $\mathscr{Q}=\operatorname{Hom}_{c}(\mathscr{K}, \mathscr{K})$. We shall consider $\mathscr{K}$ as an $\mathscr{Q}$ module. Let $\mathcal{H}_{\mathscr{K}}=\mathcal{H} \otimes \mathscr{K}$ and let $U_{\mathscr{K}}=U \otimes I$. By extension of coefficients $\mathcal{H}_{\mathscr{K}}$ is an $\mathbb{Q}$ module and $U_{\mathscr{K}}$ is an $\mathbb{Q}$-linear representation of $N$ in $\mathcal{K}_{\mathscr{K}}$. Also the scalar product $($, ) on $\mathcal{H} \times \mathcal{H}$ has a unique extension to an $\mathcal{Q}$-valued scalar product $(,)_{\mathcal{K}}$ on $\mathcal{H}_{\mathcal{K}} \times \mathcal{H}_{\mathcal{K}}$ which satisfies

$$
(c u, v)_{\mathscr{K}}=c(u, v)_{\mathscr{K}}, \quad\left(u, c^{*} v\right)_{\mathscr{K}}=(u, v)_{\mathscr{K}_{c}}
$$

for all $c \in \mathbb{Q}$ where $c^{*}$ denotes Hermitian adjoint in $\mathscr{K}$. The extension is defined by $(u \otimes a, v \otimes b)=(u, v) a \otimes b$ where $a \otimes b$ denotes the element of $\mathbb{Q}$ defined by $a \otimes b(x)=a(x, b)$.

It is easily verified that $C^{p}\left(\mathscr{P}_{0}, C\right)=C^{\infty}\left(U_{\mathscr{K}}\right)$. The following is easily verified from the corresponding fact for $\mathcal{H}$ (cf. [17]). Let $C^{\infty}\left(U_{\mathscr{K}}\right)=C^{\infty}(U) \otimes \mathscr{K}$ be denoted by $C_{\mathscr{K}}$.

Lemma 5. $v \in C^{\infty}\left(U_{\mathscr{K}}\right)$ iff $g \rightarrow\left(U_{\mathscr{K}}(g) v, w\right)_{\mathscr{K}} \in C^{\infty}(N, \mathcal{Q})$ for all $w \in \mathcal{K}_{\mathscr{K}}$.

Now, let $\mathscr{U}$ be the universal enveloping algebra of $\Re$ and let $\mathscr{U}_{\mathfrak{K}}=\mathscr{Q} \otimes \mathbb{Q}$. Let $\left(Y_{1}, \ldots, Y_{n}\right)=Y$ be an ordered basis for $\Re$ and let $A(k)$ denote the set of multi-indices $\alpha$ of length $k$ such that $1 \leqslant \alpha(1) \leqslant \alpha(2) \leqslant \cdots \leqslant \alpha(k) \leqslant n$. For each $\alpha \in A(k)$, let $Y_{\alpha}=Y_{\alpha(1)} Y_{\alpha(2)} \cdots Y_{\alpha(k)} \in \mathcal{Q}$. Every element $X$ of $\mathscr{U}_{\gamma \mathcal{K}}$ has a unique expression of the form $X=\Sigma_{k} \Sigma_{\alpha \in A(k)} Y_{\alpha} \otimes C_{\alpha}$ where $C_{\alpha} \in \mathbb{Q}$. The order of $X$ is, by definition, the largest $k$ such that $C_{\alpha} \neq 0$ for some $\alpha \in A(k)$. The symbol $\sigma(X)$ is by definition the $\mathcal{Q}$-valued polynomial on $\mathbf{R}^{n}$ defined by the following formula, where $k$ is the order of $X$.

$$
\sigma(X)(y)=\sum_{\alpha \in A(k)} C_{\alpha} y_{\alpha(1)}, \ldots, y_{\alpha(k)}
$$

where $y=\left(y_{1}, y_{2}, \ldots, y_{n}\right) \in \mathbf{R}^{n}$.

Definition. $X$ is elliptic if $\sigma(X)(y)$ is invertible as an element of the algebra $\mathbb{Q}$ for all nonzero $y \in \mathbf{R}$.

Now $U_{\mathscr{K}}$ gives rise to a mapping $\partial U_{\mathscr{K}}$ of $\mathscr{Q}_{\mathscr{K}}$ into the space of mappings of $C_{\mathscr{K}}$ into $C_{\mathscr{K}}$ defined by

$$
\partial U_{\mathscr{K}}\left(Y_{\alpha} \otimes C_{\alpha}\right)=\partial U\left(Y_{\alpha}\right) \otimes C_{\alpha} .
$$

LEMMA 6. The restriction of $\square-I$ to $C_{\mathscr{K}}$ is the image of an elliptic element $X$ of थr.

Proof. To see that $\square$ is the image of an element of $\mathscr{U}_{\mathscr{N}}$ is easy. Let an isomorphism of $\mathscr{K}$ with $\mathbf{C}^{n}$ be given. Then $\mathcal{Q}$ is just the $m \times m$ matrices with entries in $\mathbf{C}$ and $\mathscr{Q}_{\mathscr{K}}$ is the set of $m \times m$ matrices with entries in $\mathscr{U}_{c}$ and $C_{\mathscr{K}}$ is $C^{m}$. Hence any operator $A$ on $C \otimes \mathcal{K}$ which, relative to this basis, is expressible in the form $(A f)_{i}=\Sigma_{j} \partial U\left(X_{i j}\right) f_{j}$ with $X_{i j} \in \mathcal{Q}_{c}$ is in $\partial U_{\mathscr{g}}\left(\mathscr{U}_{\mathscr{F}}\right)$. If follows from the formulas in Lemma 2 that $\square$ is of this form. $-I$ is also in the image of $\mathscr{U}_{\mathcal{K}}$ for let $Z_{0}$ generate the center $\mathcal{Z}$ of $\Re$. We may choose $Z_{0}$ so that $\lambda\left(Z_{0}\right)=1$. Then $\partial U_{\mathscr{K}}\left(Z_{0}^{2}\right)=\left(i \lambda\left(Z_{0}\right)\right)^{2} I=-I$. 
Now, to show the ellipticity, recall that from Lemma 2 , on $C_{\mathscr{K}} \square=\delta \bar{\partial}+\bar{\partial} \delta$ where $\bar{\partial}=D+1 \otimes \bar{\partial}_{1}, \delta=D^{*}+1 \otimes \bar{\partial}_{1}^{*}$. (See Lemma 2 for notation.) The principal part of $\square$ is the same as that of $\square_{0}=D^{*} D+D D^{*}$. From the formulas in Lemma $2, \square_{0}$ is easily computed.

$$
\begin{aligned}
\square_{0} f\left(X_{1}, \ldots, X_{p}\right)= & -\sum \bar{E}_{i} E_{i} f\left(X_{1}, \ldots, X_{p}\right) \\
& +\sum(-1)^{j+1} \bar{E}_{i} X_{j} f\left(E_{i}, X_{1}, \ldots, X_{j}, \ldots, X_{p}\right) \\
& -\sum(-1)^{j+1} X_{j} \bar{E}_{i} f\left(E_{i}, X_{1}, \ldots, X_{j}, \ldots, X_{p}\right) \\
= & -\sum \bar{E}_{i} E_{j} f\left(X_{1}, \ldots, X_{p}\right) \\
& +\sum(-1)^{j+1}\left[\bar{E}_{i}, X_{j}\right] f\left(E_{i}, X_{1}, \ldots, X_{j}, \ldots, X_{p}\right)
\end{aligned}
$$

where $E_{1}, \ldots, E_{n}$ is a basis of $\mathscr{P}_{0}$ and - denotes conjugation in $\mathscr{N}_{c}$. The principal part is the image under $\partial U_{\mathscr{K}}$ of $-\sum \bar{E}_{i} E_{i} \otimes I$. To compute the symbol let $E_{k}=$ $Y_{2 k-1}+i Y_{2 k}$ where $Y_{i} \in \mathcal{R}$. Then $\left\{Y_{i}\right\} \cup\left\{Z_{0}\right\}$ is a basis of $\Re_{0}$. Let $\left(y_{1}, \ldots, y_{2 n}, t\right) \in \mathbf{R}^{2 n+1}$. Let $z_{k}=y_{2 k-1}+i y_{2 k}$. It is easily seen that the symbol of $\square_{0}-I$ in this basis is $-\left(t^{2}+\Sigma\left|z_{i}\right|^{2}\right) I$. Q.E.D.

Our proposition is a consequence of the following more general proposition which is a generalization of results of Goodman. The proof is a straightforward mimicking of the proof given in [6]. One merely uses $Q$ as the coefficient space instead of $C$. We shall sketch the proof.

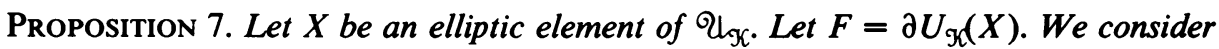
$F$ to be a partially defined operator on $\mathcal{H}_{\mathscr{H}}$. Let $\bar{F}$ be its closure in $(,)_{\mathscr{K}}$. Then $\cap_{n}$ domain $(\bar{F})^{n}=C_{\mathscr{K}}$. Furthermore the topology on $C_{\mathscr{K}}$ defined by the seminorms $x \rightarrow\left\|(\bar{F})^{n} x\right\|$ agrees with the $C_{\mathscr{K}}$ topology.

Proof. One inclusion is obvious. To prove the other let $w \in \cap$ domain $\bar{F}^{n}$ be given. Let $v \in \mathcal{K}_{\mathcal{K}}$ be fixed but arbitrary. Let $\phi(g, w)=\left(U_{\mathscr{T}}(g) v, w\right)_{\mathscr{K}}$. We shall use $\phi$ to define a distribution $\phi(w)$ on $C_{c}^{\infty}(N, \mathcal{Q})$ by setting

$$
\langle\psi, \phi(w)\rangle=\int \operatorname{tr}\left(\phi(g, w)^{*} \psi(g)\right) d g .
$$

Let $X=\Sigma_{k} \Sigma_{\alpha \in A(k)} X_{\alpha} \otimes C_{\alpha}$. We set $\tilde{F} \psi=\Sigma X_{\alpha} \psi C_{\alpha}$ where $X_{\alpha}$ acts as a right invariant operator and $\psi \in C_{c}^{\infty}(N, \mathbb{Q})$. It is easily verified that $\left\langle(\tilde{F})^{n} \psi, \phi(w)\right\rangle=$ $\left\langle\psi, \phi\left(\bar{F}^{n} w\right)\right\rangle$. It follows that $\left(\tilde{F}^{*}\right)^{n} \phi(w)=\phi\left(\bar{F}^{n} w\right)$ in the sense of distributions where “*” denotes adjoint in $C_{c}^{\infty}(N, \mathbb{Q})$. Once we have shown that $\tilde{F}$ is an elliptic system of differential operators on $C_{c}^{\infty}(N, \mathcal{Q})$, it will follow from the continuity of $\phi\left(\bar{F}^{n} w\right)$ that $\phi(w)$ is a $C^{\infty}$ function. Since $v$ was arbitrary, we will then be done.

LEMMA 8. The system $\tilde{F}$ is elliptic on $\Re$.

Proof. By right invariance it suffices to show ellipticity in a neighborhood of $e$. By continuity of the coefficients, it suffices to show ellipticity at $e$. At $e$, one picks a local coordinate system for which $Y_{i} \sim \partial / \partial x_{i}$. Q.E.D.

Now we prove Theorem 3. 
Proof. By definition the adjoint of $\delta \bar{\partial}+\bar{\partial} \delta$ extends $\square$ so $C^{p}\left(\mathscr{P}_{0}, C\right)=$ $\cap$ domain $\square^{n}$. In particular $\mathcal{H}^{p}\left(\mathscr{P}_{0}, U\right) \subset C^{p}\left(\mathscr{P}_{0}, C\right)$ and it is closed since $\square$ is continuous. $f \in \operatorname{ker} \square$ iff $\delta_{c} f=\bar{\partial}_{c} f=0$ so $\mathcal{H}^{p}\left(\mathscr{P}_{0}, U\right) \subset \operatorname{ker} \bar{\partial}$. In $C^{p}\left(\mathscr{P}_{0}, \mathcal{H}\right)$, $\mathcal{H}^{p}\left(\mathscr{P}_{0}, U\right)^{\perp}=[\operatorname{Im} \bar{\partial}] \oplus[\operatorname{Im} \delta]$ so $C^{p}\left(\mathscr{P}_{0}, \mathcal{H}\right)=\mathcal{H}^{p}\left(\mathscr{P}_{0}, U\right) \oplus[\operatorname{Im} \bar{\partial}] \oplus[\operatorname{Im} \delta]$. $\left(\mathcal{H}^{P}\left(\mathscr{P}_{0}, U\right)\right.$ is closed in $C^{p}\left(P_{0}, \mathcal{H}\right)$ since ker $\bar{\partial}_{c}$ and ker $\delta_{c}$ are both clearly closed.)

This is an orthogonal decomposition since $\operatorname{Im} \bar{\partial} \subset \operatorname{ker} \bar{\partial}_{c} \subset(\operatorname{Im} \delta)^{\perp}$. Hence it also follows that ker $\bar{\partial} \subset \mathcal{H}^{p}\left(\mathcal{P}_{0}, \mathcal{H}\right) \oplus[\operatorname{Im} \bar{\partial}]$.

To prove our theorem we introduce a chain of Hilbert spaces. Let $(,)_{p, k}$ be the scalar product structure on $C^{p}\left(\mathscr{P}_{0}, C\right)$ defined by $(v, w)_{p, k}=\sum_{j=0}^{k}\left(\square^{j} v, \square^{j} w\right)_{p}$. Let $\mathcal{H}(p, k)$ be the completion of $C^{p}\left(\mathscr{P}_{0}, C\right)$ in the corresponding norm and let []$_{k}$ denote closure in $\mathcal{H}(p, k)$. Clearly, we may consider $\mathcal{H}(p, k) \supset \mathcal{H}\left(p, k^{\prime}\right)$ for $k \geqslant k^{\prime}$.

LeMma 9. Let $C_{0}$ be a subspace of $C^{p}\left(\mathscr{P}_{0}, C\right)$. Then $\left[C_{0}\right]_{\infty}=\bigcap_{k}\left[C_{0}\right]_{k}$.

Proof. This follows easily from Proposition 7. Q.E.D.

Now, $\delta$ and $\bar{\partial}$ clearly commute with $\square$ on $C^{p}\left(\mathscr{P}_{0}, C\right)$. Hence $\delta$ and $\bar{\partial}$ are adjoint in $(,)_{p, k}$. It follows as above that ker $\bar{\partial} \subset \mathcal{F}^{p}\left(\mathscr{P}_{0}, \mathcal{H}\right) \oplus[\operatorname{Im} \bar{\partial}]_{k}$. Taking intersections we see ker $\bar{\partial} \subset \mathscr{H}^{p}\left(\mathscr{P}_{0}, \mathcal{H}\right) \oplus[\operatorname{Im} \bar{\partial}]_{\infty}$. Since $\bar{\partial}$ is continuous in $C^{p}\left(\mathscr{P}_{0}, C\right)$, the reverse inclusion is obvious. Q.E.D.

The proof of Theorem 3 is now complete.

III. Computation of cohomology. In this section we prove our main results. According to Kirillov theory there is a one-to-one correspondence between orbits of the coadjoint representation of $N$ in $\Re^{*}$ and elements of $\hat{N}$. Let $U^{\lambda}$ be any irreducible unitary representaton of $N$ corresponding to $\lambda$. It is easily seen that for $z \in Z, U^{\lambda}(z)=\chi(z) I$ where $\chi$ is as in $\S$ I. Let $U^{-\lambda}$ be the adjoint representation to $U^{\lambda}$.

Now recall that $T^{p}$ is the representation defined from the left action of $N$ on $\mathcal{H}^{p}(E) . T^{p}$ transforms according to $\bar{\chi}$ on $Z$. Our first main result of this section is the following "Frobenius" reciprocity theorem, which does not use the abelian assumption on $\mathscr{P}_{0}$.

THEOREM 10. $\mathcal{H}^{p}(E)$ is nontrivial iff $\mathcal{H}^{p}\left(\mathscr{P}_{0}, C^{\lambda}\right)$ is nontrivial where $C^{\lambda}=$ $C^{\infty}\left(U^{\lambda}\right)$. Furthermore, $T^{p}$ is primary and quasi-equivalent to $U^{-\lambda}$. The multiplicity of $U^{-\lambda}$ in $T^{p}$ is the dimension of $\mathcal{H C}^{p}\left(\mathscr{P}_{0}, C^{\lambda}\right)$.

Proof. We shall prove the second statement first.

LEMMA 11. $U^{\lambda}$ is square-integrable in the sense of Moore-Wolf [13].

Proof. It suffices from [13] to show that the radical of the form $B_{\lambda}(x, y)=$ $\lambda([x, y])$ is $\mathcal{Z}$. But $B_{\lambda}(x, y)=0$ for all $y \in \Re$ implies $B_{\lambda}\left(x, \Re_{c}\right)=0$ and hence $x \in \mathscr{P}$ since $\mathcal{P}$ is maximal subordinate. Hence $x \in \mathscr{P} \cap \mathcal{X}$. Q.E.D.

The second statement of our theorem now follows for $U^{-\lambda}$ is also square integrable. From Moore-Wolf [13], $U^{-\lambda}$ is determined up to unitary equivalence by its restriction to $Z$. Since every irreducible representation occurring in any direct integral decomposition agrees with $U^{-\lambda}$ on $Z$, the primarity of $T^{p}$ follows. 
The first statement follows from the second and third so we need only prove the third. Let $I\left(U^{-\lambda}, T^{p}\right)$ be the intertwining algebra from $U^{-\lambda}$ to $T^{p}$. The dimension of this algebra is the multiplicity of $U^{-\lambda}$ in $T^{p}$. $U^{\lambda}$ is equivalent with $U^{-\lambda}$ under a conjugate linear intertwining operator so $\operatorname{dim} I\left(U^{-\lambda}, T^{p}\right)=\operatorname{dim} I^{*}\left(U^{\lambda}, T^{p}\right)$ where $I^{*}$ is the conjugate linear intertwining algebra.

LEMMA 12. $I^{*}\left(U^{\lambda}, T^{p}\right)$ and $\mathcal{H}^{p}\left(\mathscr{P}_{0}, U^{\lambda}\right)$ are canonically isomorphic.

Proof. Let $X=\left(X_{1}, X_{2}, \ldots, X_{p}\right) \in \overline{\mathcal{P}}_{0}^{p}$. Let $\varepsilon(X)$ be the linear functional on $\mathcal{H}\left(T^{p}\right)=\mathcal{H}^{p}\left(\mathscr{P}_{0}, U^{x}\right)$ defined by $\langle f, \varepsilon(X)\rangle=f\left(e, \bar{X}_{1}, \ldots, \bar{X}_{p}\right) . \varepsilon(X)$ is continuous in the $C^{\infty}\left(U^{X}\right)$ topology on $\mathcal{F}^{P}\left(\mathscr{P}_{0}, U^{X}\right)$. By Theorem 2, this topology is the same as the Hilbert topology on $\mathcal{H}^{p}\left(\mathscr{P}_{0}, U^{x}\right)$ so there is a vector $\varepsilon(X)$ in $\mathcal{H}\left(T^{p}\right)$ such that $\langle f, \varepsilon(X)\rangle=(f, \varepsilon(X))_{p}$. The mapping $X \rightarrow \varepsilon(X)$ is a complex, $p$-linear mapping of $\left(\overline{\mathscr{P}}_{0}\right)^{p}$ into $\mathcal{K}\left(T^{p}\right)$. We shall consider $\varepsilon$ as an element of $C^{p}\left(\overline{\mathscr{P}}_{0}, \mathcal{H}\left(T^{p}\right)\right)$.

LEMMA 13. $\varepsilon \in \mathcal{H}^{p}\left(\overline{\mathscr{P}}_{0}, T^{p}\right)$.

Proof. First we must show that $\varepsilon \in C^{p}\left(\mathscr{P}_{0}, T^{p}\right)$. But for all $Y \in \Re, f \in$ $C^{\infty}\left(T^{p}\right)$ and $X_{1}, \ldots, X_{p} \in\left(\overline{\mathcal{P}}_{0}\right)^{p},\left(\partial T^{p}(Y) f, \varepsilon\left(X_{1}, \ldots, X_{p}\right)\right)=-Y f\left(e, \bar{X}_{1}, \ldots, \bar{X}_{p}\right)$ where $Y$ acts as a right invariant differential operator. But $e$ is central so $-Y f\left(e, \bar{X}_{1}, \ldots, \bar{X}_{p}\right)=-\partial U^{x}(Y) f\left(e, \bar{X}_{1}, \ldots, \bar{X}_{p}\right)$. Hence

$$
\begin{aligned}
\left(\partial T^{p}(Y) f, \varepsilon\left(X_{1}, \ldots, X_{p}\right)\right) & =\left(-\partial U^{x}(Y) f, \varepsilon\left(X_{1}, \ldots, X_{p}\right)\right) \\
& =\left(f, \partial U^{x}(\bar{Y}) \varepsilon\left(X_{1}, \ldots, X_{p}\right)\right) .
\end{aligned}
$$

The latter expression exists because

$$
\varepsilon\left(X_{1}, \ldots, X_{p}\right) \in \mathcal{F}^{p}\left(\mathscr{P}_{0}, U^{x}\right) \subset C^{p}\left(\mathscr{P}_{0}, C^{x}\right) .
$$

Hence $\varepsilon\left(X_{1}, \ldots, X_{p}\right)$ has weak derivatives of all orders so $\varepsilon\left(X_{1}, \ldots, X_{p}\right) \in$ $C^{\infty}\left(T^{p}\right)$ as desired. To show the harmonicity, let $f \in C^{\infty}\left(T^{p}\right)$. Then, using the centrality of $e$ as above, $\left(f, \bar{\partial} \varepsilon\left(X_{1}, \ldots, X_{p+1}\right)\right)=\bar{\partial} f\left(e, \bar{X}_{1}, \ldots, \bar{X}_{p+1}\right)=0$ for all $X_{1}, \ldots, X_{p+1} \in\left(\overline{\mathscr{P}}_{0}\right)^{p+1}$ where the $\bar{\partial}$ on the left corresponds to the $\bar{\partial}$ operator in the complex $C^{p}\left(\mathscr{P}_{0}, C^{\infty}\left(T^{p}\right)\right)$ and the one on the right is relative to $C^{p}(E)$. Hence $\bar{\partial} \varepsilon=0$. Similarly, using the formulas in Lemma 1 , we show that $\left(f, \delta \varepsilon\left(X_{1}, \ldots, X_{p+1}\right)\right)=\delta f\left(e, \bar{X}_{1}, \ldots, \bar{X}_{p+1}\right)=0$ for all $f \in \mathcal{H}^{p}\left(\mathscr{P}_{0}, U^{\lambda}\right)$ where the first occurrence of $\delta$ is the $C^{p}\left(\widehat{\mathscr{P}}_{0}, C^{\infty}\left(T^{p}\right)\right) \delta$ and the second is the $C^{p}\left(\mathscr{P}_{0}, C^{x}\right) \delta$. Hence $\delta \varepsilon=0$, proving the lemma. Q.E.D.

Now, let $A \in I^{*}\left(U^{x}, T^{p}\right)$ and let $A^{*}$ be the Hermitian adjoint of $A$ (i.e. $\left.(A v, w)=\left(v, A^{*} w\right)^{-}\right)$. We extend $A^{*}$ to a mapping of $C^{q}\left(\overline{\mathcal{P}}_{0}, \mathcal{H}\left(T^{p}\right)\right)$ into $C^{q}\left(\mathscr{P}_{0}, \mathcal{H}\left(U^{x}\right)\right)$ by setting $\left(A^{*} g\right)\left(X_{1}, \ldots, X_{q}\right)=A^{*}\left(g\left(\bar{X}_{1}, \ldots, \bar{X}_{q}\right)\right)$. Let $\phi_{A} \in$ $C^{p}\left(\mathscr{P}_{0}, \mathcal{H}\left(U^{x}\right)\right)$ be $\phi_{A}=A^{*} \varepsilon$. It is clear that $\phi_{A} \in \mathcal{H}^{p}\left(\mathscr{P}_{0}, U^{x}\right)$.

LeMma 14. The mapping $A \rightarrow \phi_{A}$ is a 1-1 conjugate linear mapping of $I^{*}\left(U^{x}, T^{p}\right)$ onto $\mathcal{H}^{p}\left(\mathscr{P}_{0}, U^{x}\right)$. 
Proof. The 1-1 property is easily seen for suppose $A^{*} \varepsilon=0$. Then for all $f \in C^{x}$, $X_{i} \in \overline{\mathscr{P}}_{0},\left(A f, \varepsilon\left(\bar{X}_{1}, \ldots, \bar{X}_{p}\right)\right)=0=A f\left(e, X_{1}, \ldots, X_{n}\right)$ but then

$$
\begin{aligned}
A f\left(x, \bar{X}_{1}, \ldots, \bar{X}_{n}\right) & =T^{p}\left(x^{-1}\right) A f\left(e, \bar{X}_{1}, \ldots, \bar{X}_{n}\right) \\
& =\left(A U\left(x^{-1}\right) f\right)\left(e, \bar{X}_{1}, \ldots, \bar{X}_{n}\right)=0 .
\end{aligned}
$$

Hence $A f=0$ showing that $A=0$.

To prove the surjectivity, let $\phi \in \mathcal{F}^{p}\left(\mathscr{P}_{0}, U^{\lambda}\right)$. For $\left(X_{1}, \ldots, X_{p}\right) \in \mathscr{P}_{0}^{p}$, and each $v \in \mathcal{H}\left(U^{\lambda}\right)$, let $A_{\phi}(v)\left(x, X_{1}, \ldots, X_{p}\right)=\left(U^{\lambda}\left(x^{-1}\right) v, \phi\left(X_{1}, \ldots, X_{p}\right)\right)^{-}$. Since $U^{\lambda}$ is square integrable modulo $Z$ (Lemma 11), $A_{\phi}(v)$ is square integrable as a function of $x$. In fact, it is easily seen that for $X_{1}, \ldots, X_{p}$ fixed, $A_{\phi}(v)$ is an element of $C^{x}$. A straightforward computation shows that $A_{\phi}(v) \in \mathcal{F}^{p}\left(\overline{\mathscr{P}}_{0}, U^{x}\right)$ and $A_{\phi}$ is an intertwining operator in $I^{*}\left(U^{\lambda}, T^{p}\right)$. Clearly $A_{\phi}^{*} \varepsilon=\phi$ so $\phi \rightarrow A_{\phi}$ is the inverse of $A \rightarrow \phi_{A}$. Q.E.D.

We now begin the proof of our main result. Let $\phi$ be the form on $\mathscr{P}_{0} \times \mathscr{P}_{0}$ defined by $\phi(x, y)=-i \lambda([x, y]) . \phi$ is Hermitian symmetric, and nondegenerate, although $\phi$ is not usually positive. There is a number $p$ and a basis $E_{1}, \ldots, E_{n}$ of $\mathscr{P}_{0}$ such that

$$
\phi\left(E_{i}, E_{j}\right)=0, \quad i \neq j, \quad \phi\left(E_{i}, E_{i}\right)= \pm 1,
$$

where the plus sign prevails iff $i \leqslant p$. The number $p$ is called the signature of $\phi$. Our main result is

THEOREM 15. $\mathcal{H}^{p}(E)$ is nontrivial iff $p=$ signature $\phi$. In this case $T^{p}$ is irreducible.

The above results reduce us to consideration of $\underline{H}^{q}\left(\mathscr{P}_{0}, C^{\lambda}\right)$. Note that if the image of $\bar{\partial}$ is closed, $\underline{H}^{q}\left(\mathscr{P}_{0}, C^{\lambda}\right)=H^{q}\left(\mathscr{P}_{0}, C^{\lambda}\right)$. This will be the case iff $H^{q}\left(\mathscr{P}_{0}, C^{\lambda}\right)$ is Hausdorff in its natural topology. Hence the above theorem is implied by the following.

THEOREM 16. $H^{p}\left(\mathscr{P}_{0}, C^{\lambda}\right)$ is nontrivial iff $p=$ signature $\phi$. In this case $H^{P}\left(\mathscr{P}_{0}, C^{\lambda}\right)$ is Hausdorff and one dimensional.

We shall use the Hochschild-Serre spectral sequence. Let $\Re_{0}$ be a complex subalgebra of $\Re_{c}$ and let $M$ be an $\Re_{0}$ module. Let $\mathscr{K} \subset \Re_{0}$ be a subalgebra and let

$$
M^{\mathscr{X}}=\{x \in M \mid y \cdot x=0 \text { for all } y \in \mathscr{K}\} .
$$

The cohomology spaces $H^{p}(\mathcal{K}, M)$ are defined by the same formulas that $H^{p}\left(\mathscr{T}_{0}, C\right)$ was defined in $\S \mathrm{I}$. If $\mathscr{K}$ is an ideal of $\mathscr{T}_{0}$, the space $C^{p}(\mathscr{K}, M)$ is an $\Re_{0}$ module with the action being defined by

$$
\begin{aligned}
(X \cdot f)\left(X_{1}, \ldots, X_{p}\right)= & X \cdot f\left(X_{1}, \ldots, X_{p}\right) \\
& -\sum_{i=1}^{k} f\left(X_{1}, \ldots,\left[X, X_{i}\right], \ldots, X_{p}\right) .
\end{aligned}
$$

This action factors to an action of $\mathscr{T}_{0}$ on $H^{p}(\mathscr{K}, M) . \mathscr{K}$ acts trivially so $H^{p}(\mathscr{K}, M)$ is in fact an $\mathscr{K}_{0} / \mathcal{K}$ module. The Hochschild-Serre spectral sequence theorem is the following 
THEOREM 17. Let $m \geqslant 1$. If $m>1$, assume that $H^{n}(\mathcal{K}, M)=0$ for $2<n \leqslant m$. Then there is an exact sequence of homomorphisms

$$
\begin{aligned}
H^{m}\left(\Re_{0} / \mathcal{K}, M^{\mathscr{K}}\right) & \stackrel{l_{m}}{\rightarrow} H^{m}\left(\Re_{0}, M\right) \stackrel{r_{m}^{\prime}}{\rightarrow} H^{m-1}\left(\Re_{0} / \mathcal{K}, H^{1}(\mathcal{K}, M)\right) \\
& \stackrel{d_{2}^{\prime}}{\rightarrow} H^{m+1}\left(\Re_{0} / \mathcal{K}, M^{\mathscr{K}}\right) \stackrel{l_{m+1}}{\rightarrow} H^{m+1}\left(\Re_{0}, M\right) .
\end{aligned}
$$

We shall apply this theorem only in the case that $\operatorname{dim} \mathscr{K}=1$ so that the condition on $H^{n}(\mathcal{K}, M)$ is always satisfied. It will also always be the case that either (i) $M^{\mathscr{K}}=0$ or (ii) $H^{1}(\mathscr{K}, M)=0$.

It follows from Hochschild-Serre that in case (i), $H^{m}\left(\Re_{0}, M\right)$ and $H\left(\Re_{0} / \mathcal{K}, H^{1}(\mathcal{K}, M)\right)$ are isomorphic for $m>1$ and in case (ii), $H^{m}\left(\Re_{0}, M\right)$ is the image of $H^{m}\left(\Re_{0}, M^{\Re}\right)$ under $l_{m}$ for $m>1$ with this mapping being an isomorphism for $m \geqslant 2$. (Actually the mapping is an isomorphism for $m=1$ also. This follows from Theorem 6 of [8].)

Suppose $M$ is a topological vector space which is an $\Re_{0}$ module. We topologize $C^{p}\left(\Re_{0}, M\right)$ and $H^{p}\left(\Re_{0}, M\right)$ in the obvious manner. The following lemma is our main tool in keeping track of the Hausdorff property.

Lemma 18. Suppose $\mathscr{K}$ is a one dimensional ideal of $\Re_{0}$ and $\Re_{0}$ is abelian. Then the mappings $l_{m}$ and $r_{m}^{\prime}$ above are continuous. Also, suppose $H^{1}(\mathcal{K}, M)$ is zero and there exists a continuous mapping $T: Z^{1}(\mathscr{K}, M) \rightarrow C^{0}(\mathcal{K}, M)$ such that $\bar{\partial} T=I$ where $I$ is the identity on $Z^{1}(\mathcal{K}, M)$. Then there is a continuous mapping $s_{m}$ : $H^{m}\left(\Re_{0}, M\right) \rightarrow H^{m}\left(\Re_{0} / \mathscr{K}, M^{\mathscr{K}}\right)$ such that $l_{m} s_{m}=I$.

Proof. Let $r_{m}$ be the mapping of $C^{m}\left(\mathscr{T}_{0}, M\right)$ into $C^{m-1}\left(\mathscr{T}_{0}, C^{1}(\mathcal{K}, M)\right)$ given by restriction of the first variable to $\mathcal{K}$. Then for $f \in C^{m}\left(\mathscr{T}_{0}, M\right), r_{m} f$ is zero whenever any of its $\Re_{0}$ arguments lie in $\mathscr{K}$, from the one dimensionality of $\mathcal{K}$. Hence $r_{m} f$ defines an element of $C^{m-1}\left(\Re_{0} / \mathscr{K}, C^{1}(\mathcal{K}, M)\right), C^{1}(\mathcal{K}, M)=$ $Z^{1}(\mathcal{K}, M)$ by one dimensionality so we may project $r_{m} f$ to an element of $C^{m-1}\left(\Re_{0} / \mathcal{K}, H^{1}(\mathcal{K}, M)\right)$. It is easily verified that the element so defined is a cocycle. The mapping $r_{m}^{\prime}$ is obtained by further projecting $r_{m} f$ to $H^{m-1}\left(\Re_{0} / \mathscr{K}, H^{1}(\mathscr{K}, M)\right)$. Clearly $r_{m}^{\prime}$ is then continuous.

The mapping $l_{m}$ is defined by naturality from the lifting mapping of $C^{m}\left(\mathscr{T}_{0} / \mathscr{K}, M^{\mathscr{K}}\right)$ into $C^{m}\left(\mathscr{T}_{0}, M\right)$ so it is continuous. To define $s_{m}$, let $T$ be as hypothesized above. For $f \in C^{m}\left(\Re_{0}, M\right)$, let $S f \in C^{m-1}\left(\Re_{0}, M\right)$ be defined by

$$
S f\left(X_{1}, \ldots, X_{m-1}\right)=T\left(f\left(\cdot, X_{1}, \ldots, X_{m-1}\right) \mid \mathcal{K}\right) .
$$

Note that if any $X_{i} \in \mathscr{K}$, then $S f\left(X_{1}, \ldots, X_{m-1}\right)=0$. We claim that $I-\bar{\partial} S$ maps $Z^{m}\left(\Re_{0}, M\right)$ into $Z^{m}\left(\Re_{0}, M\right)$. Furthermore, we claim that if $f \in Z^{m}\left(\Re_{0}, M\right)$, then $(I-\bar{\partial} S) f \in C^{m}\left(\mathscr{N}_{0} / \mathscr{K}, M^{\mathscr{K}}\right)$. The first claim is obvious. To see the second note first that for any $K \in \mathscr{K}$,

$$
\begin{aligned}
K(I-\bar{\partial} S f)\left(X_{1}, \ldots, X_{m}\right)= & K f\left(X_{1}, \ldots, X_{m}\right) \\
& -\sum(-1)^{i+1} X_{i} K S f\left(X_{1}, \ldots, X_{i}, \ldots, X_{m}\right) \\
= & \bar{\partial} f\left(K, X_{1}, \ldots, X_{m}\right)=0 .
\end{aligned}
$$


Furthermore, if $X_{1} \in \mathscr{K}$,

$$
(I-\bar{\partial} S) f\left(X_{1}, \ldots, X_{m}\right)=f\left(X_{1}, \ldots, X_{m}\right)-X_{1} S f\left(X_{2}, \ldots, X_{m}\right)=0 .
$$

This shows the second claim.

The mapping $f \rightarrow I-\bar{\partial} S f$ projects to the described mapping $s_{m}$. Q.E.D.

COROLlARY 19. Under the first hypothesis of the above lemma, $H^{m}\left(\Re_{0}, M\right)$ will be Hausdorff if $H^{m-1}\left(\Re_{0} / \mathcal{K}, H^{1}(\mathcal{K}, M)\right)$ is Hausdorff. Under the second hypothesis, $H^{m}(\mathscr{N}, M)$ will be Hausdorff if $H^{m}\left(\mathscr{T}_{0} / \mathscr{K}, M^{\mathscr{K}}\right)$ is Hausdorff.

Now, let us begin the proof of Theorem 16 above.

We shall consistently identify $\Re$ and $N$ by means of the exponential mapping so that multiplication in $N$ is given by the Campbell-Hausdorff formula.

Let $J: \mathscr{N}_{c} \rightarrow \mathscr{\Re}_{c}$ be the unique complex linear mapping such that $\mathscr{P}_{0}$ is the $+i$ eigenspace of $J, \widehat{\mathscr{P}}_{0}$ is the $-i$ eigenspace, and $\mathscr{\Psi}_{c}$ is the +1 eigenspace. It is easily seen that

(i) $J^{4}=I$,

(ii) $J(\mathfrak{x}) \subset \Re$,

(iii) $[X, Y]=[J X, J Y]$ for all $X, Y \in \Re$.

Part (iii) above is a simple consequence of the abelian property of $\mathcal{P}$. (iii) is in fact equivalent to the commutativity of $\mathscr{P} . \mathscr{P}$ is then the image of $\Re_{c}$ under $I-i J$.

Case I. $[\Re, \mathscr{N}]=\mathscr{Z}$. Let $E_{1}, \ldots, E_{n}$ be a fixed complex basis of $\mathscr{P}_{0}$ which is orthonormal with respect to $\phi$ as described at the beginning of this section. Let $E_{j}=\left(X_{j}-i Y_{j}\right) / 2$ where $X_{i}, Y_{j} \in \mathcal{N}$ and $Y_{j}=J X_{j}$. Let $Z_{0} \in \mathscr{Z}$ be such that $\lambda\left(Z_{0}\right)=1$. Then $\left\{Z_{0}, X_{1}, \ldots, X_{n}, Y_{1}, \ldots, Y_{n}\right\}$ is a basis of $\Re_{0}$. From the equality $-i \lambda\left(\left[E_{i}, \bar{E}_{j}\right]\right)= \pm \delta_{i j}$, it is easily verified that

(i) $\left[X_{i}, X_{j}\right]=0=\left[Y_{i}, Y_{j}\right]$,

(ii)

$$
\begin{aligned}
{\left[X_{i}, Y_{j}\right] } & =\frac{1}{2} \delta_{i j} Z_{0} \quad \text { if } i \leqslant p, \\
& =-\frac{1}{2} \delta_{i j} Z_{0} \text { if } i>p .
\end{aligned}
$$

We identify $\Re$ with $\mathbf{R}^{n} \times \mathbf{R}^{n} \times \mathbf{R}$ by mapping $(x, y, t) \rightarrow\left(\sum x_{i} X_{i}+y_{i} Y_{i}\right)+t Z_{0}$ for $x=\left(x_{i}\right), y=\left(y_{i}\right) \in \mathbf{R}^{n}$ and $t \in \mathbf{R}$. Let $\mathfrak{N}$ be the subalgebra $\mathfrak{N}=\{(o, y, t)\}$. Let $\eta$ be the character of $\mathfrak{N}$ given by $\eta(o, y, t)=e^{i t}=\exp i$.

The group law on $\Re$ is defined by $u \cdot v=u+v+\frac{1}{2}[u, v] . \eta$ is a group-theoretic character and the representation $U^{\eta}=\operatorname{ind}(\Re, N, \eta)$ is irreducible and equivalent with $U^{\lambda}$.

By restriction to $\mathbf{R}^{n} \times\{0\} \times\{0\}$ in $\mathscr{N}$, we realize $U^{\eta}$ in $L^{2}\left(\mathbf{R}^{n}\right)$ as usual.

It is well known that $C^{\eta}=C^{\infty}\left(U^{\eta}\right)$ is topologically equal to $\delta\left(R^{n}\right)$, the Schwartz space of rapidly decreasing $C^{\infty}$ functions. Also

$$
\begin{aligned}
\partial U^{\eta}\left(\left(X_{j}-i Y_{j}\right)\right) f(x) & =\left(\partial / \partial x_{j}+x_{j} / 2\right) f(x), & & j<p, \\
& =\left(\partial / \partial x_{j}-x_{j} / 2\right) f(x), & & j>p .
\end{aligned}
$$


Let us first suppose $p<n$. Let $\mathscr{K}$ be the subspace of $\mathscr{P}_{0}$ spanned by $X_{n}-i Y_{n}$ over $\mathbf{C}$.

LEMMA 20. $\left(C^{\eta}\right)^{\mathscr{K}}=0$ and the image $\mathscr{K} C^{\eta}$ of $C^{\eta}$ under $\mathscr{K}$ is closed in $C^{\eta}$.

Proof. $\left(C^{\eta}\right)^{\mathscr{K}}$ is the kernel of $\partial / \partial x_{n}-x_{n} / 2$ in $\delta\left(\mathbf{R}^{n}\right)$. However, in $C^{\infty}\left(\mathbf{R}^{n}\right)$, the kernel of this operator is spanned by functions $\left(\exp +x_{n}^{2} / 4\right) c(x)$ where $c$ is independent of $x_{n}$. Such a function is never Schwartz unless it is zero.

To calculate the image we use the following sublemma, which implies the closedness of the image.

Sublemma 21. A Schwartz function $g$ is contained in the image of $\partial / \partial x_{n}-x_{n} / 2$ iff

$$
\int_{-\infty}^{\infty}\left(\exp -x_{n}^{2} / 4\right) g\left(x_{1}, \ldots, x_{n}\right) d x_{n}=0
$$

for all $\left(x_{1}, \ldots, x_{n-1}\right) \in \mathbf{R}^{n-1}$.

Proof. One implication follows by integration by parts. To prove the other implication, set

$$
f\left(x_{1}, \ldots, x_{n}\right)=\left(\exp x_{n}^{2} / 4\right) \int_{-\infty}^{x_{n}}\left(\exp -s^{2} / 4\right) g\left(x_{1}, \ldots, x_{n-1}, s\right) d s .
$$

We claim that $f$ is a Schwartz function. Clearly it suffices to fix $x_{1}, \ldots, x_{n-1}$ and consider only $x_{n}$. Let $h\left(x_{n}\right)$ be the integral in the above expression so $f\left(x_{n}\right)=$ $\left(\exp x_{n}^{2} / 4\right) h\left(x_{n}\right)$. Then $\lim _{x_{n} \rightarrow \infty} h\left(x_{n}\right)=0$. By l'Hôpital's rule, $\lim _{x_{n} \rightarrow \infty} f\left(x_{n}\right)=$ $\lim _{x_{n} \rightarrow \infty} h^{\prime}\left(x_{n}\right) /\left(-x_{n} / 2 \exp -x_{n}^{2} / 4\right)=0$. By induction and l'Hôpital's rule $\lim _{x_{n} \rightarrow \infty}\left(x_{n}\right)^{k} f\left(x_{n}\right)=0$ for all integers $k$. Similar statements hold for $-\infty$ so $\left(x_{n}\right)^{k} f\left(x_{n}\right)$ is bounded. From the Leibnitz rule for differentiating products and induction, $f^{(m)}=p_{m} f+q_{m} g_{m}$ where $p_{m}$ and $q_{m}$ are polynomials and $g_{m}$ is a Schwartz function. Hence $\left(x_{n}\right)^{k} f^{m}$ is bounded. Clearly $g=\left(\partial / \partial x_{n}+x_{n} / 2\right) f$. Q.E.D.

Next we shall compute $H^{1}\left(\mathcal{K}, C^{\eta}\right)$. Since $\mathcal{K}$ is one dimensional, $C^{1}\left(\mathcal{K}, C^{\eta}\right)$ is isomorphic with $C^{\eta}$ and $H^{1}\left(\mathcal{K}, C^{\eta}\right)$ is $C^{\eta} / \mathcal{K} C^{\eta}$. For $f \in C^{\eta}$, let $\tau f \in \mathcal{S}\left(\mathbf{R}^{n-1}\right)$ be defined by

$$
\tau f(x)=\int_{-\infty}^{\infty} f\left(x_{1}, \ldots, x_{n-1}, s\right) \exp \left(-s^{2} / 4\right) d s
$$

From the above sublemma, the kernel of $\tau$ is the image of $C^{\eta}$ under $\mathscr{K}$ so $H^{1}\left(\mathscr{K}, C^{\eta}\right) \approx$ image $\tau=S\left(\mathbf{R}^{n-1}\right) . \tau$ is obviously a topological isomorphism.

Now from Hochschild-Serre,

$$
H^{q}\left(\mathscr{P}_{0}, C^{\eta}\right) \approx H^{q-1}\left(\mathscr{P}_{0} / \mathscr{K}, H^{1}\left(\mathcal{K}, C^{\eta}\right)\right) \text {. }
$$

Using $\tau$ to identity $H^{1}\left(\mathscr{K}, C^{\eta}\right)$ with $\mathcal{S}\left(\mathbf{R}^{n-1}\right)$, we see that $\mathscr{P}_{0} / \mathscr{K}$ is spanned by the operators $\partial / \partial x_{j} \pm \frac{1}{2} x_{j}$ for $1 \leqslant j \leqslant n-1$. Let $\Re_{1}=\operatorname{span}\left\{X_{i}, Y_{j}, Z_{0} \mid i<n-1\right.$, $j<n-1\} . \mathscr{P}_{1}=\mathscr{P}_{0} \cap\left(\mathscr{T}_{1}\right)_{c}$. Then $N_{1}$ is a subalgebra of $N$ and obviously we have

$$
H^{q-1}\left(\mathscr{P}_{0} / \mathcal{K}, H^{1}\left(\mathcal{K}, C^{\eta}\right)\right) \approx H^{q-1}\left(\mathscr{P}_{1}, C^{\eta_{1}}\right)
$$

where $C^{\eta_{1}}$ is defined relative to $\Re_{1}$ as $C^{\eta}$ was defined relative to $\Re$. We conclude by induction that $H^{q-1}\left(\mathscr{P}_{0} / \mathcal{K}, H^{1}\left(\mathcal{K}, C^{\eta}\right)\right)$ is zero if $q-1 \neq n-1-p$ and that 
this space is one dimensional and Hausdorff if $q-1=n-1-p$. This implies that $H^{q}\left(\mathscr{P}_{0}, C^{\eta}\right)$ is Hausdorff and satisfies the requirements of the lemma.

Now assume $p=n$.

Sublemma 22. The operator $\partial / \partial x+x / 2$ is surjective on $\mathcal{S}(\mathbf{R})$.

Proof. Let $f \in \mathcal{S}(\mathbf{R})$ and let

$$
g(x)=e^{-x^{2} / 4} \int_{0}^{x} e^{t^{2} / 4} f(t) d t
$$

Then $f$ is the image of $g$ under the above operator. It suffices to show that $g$ is Schwartz. This follows easily from l'Hôpital's rule as in the proof of the sublemma above. Q.E.D.

Let $\mathscr{K}=\operatorname{span}_{C}\left\{\partial / \partial x_{1}+x_{1} / 2\right\}$. It follows from the above sublemma, that $H^{1}\left(\mathcal{K}, C^{\eta}\right)=0$. Hence, from Hochschild-Serre, if $q \geqslant 1$, there is a surjective mapping of $H^{q}\left(\mathscr{P}_{0} / \mathscr{K},\left(C^{\eta}\right)^{\mathscr{K}}\right)$ onto $H^{q}\left(\mathscr{P}_{0}, C^{\eta}\right)$. The space $\left(C^{\eta}\right)^{\mathscr{K}}$ is the space of functions $f$ of the form $f(x)=e^{-x_{1}^{2} / 4} g(x)$ where $g \in \mathcal{S}\left(\mathbf{R}^{n-1}\right)$. The mapping $f \rightarrow g$ defines an isomorphism between $H^{q}\left(\mathscr{P}_{0} / \mathcal{K},\left(C^{\eta}\right)^{\mathscr{K}}\right)$ and $H^{q}\left(\mathscr{P}_{1}, C^{\eta_{1}}\right)$ as above. By induction then $H^{q}\left(\mathscr{P}_{0}, C^{\eta}\right)=0$ if $q \geqslant 1 . H^{0}\left(\mathscr{P}_{0}, C^{\eta}\right)$ is just the common kernel of the operators $\partial / \partial x_{i}+x_{j} / 2$ which is simply the one-dimensional space spanned by the function $e^{-\left(x_{1}^{2}+\cdots+x_{n}^{2}\right) / 4}$.

Case II. Now suppose that $[\Re, \Re] \neq \mathscr{Z}$. We may suppose $[\Re, \Re] \neq 0$ as the theorem is obvious in the abelian case. Let $\left.\mathscr{I}_{2}=\{X \in \mathfrak{N} \| X, \Re] \subset \mathscr{Z}\right\}$. Then there is an $X \in[\Re, \mathscr{N}] \cap \mathscr{Z}_{2}-\mathscr{Z}$. The properties of $J$ ((i)-(iii) above) imply that $J X=Y \in \mathscr{Z}_{2}-\mathscr{Z} .[X, Y]=0$ since $\left[[\Re, \Re], \mathscr{L}_{2}\right]=0$.

Let $\Re_{0}$ be the subalgebra of $\mathscr{N}$ spanned by $X, Y$ and $\mathscr{Z}$. Let $\Re_{0} \supset \Re_{0}$ be the centralizer of $\Re_{0}$ in $\Re$. Then $\Re_{0}$ can also be described as the orthogonal set of $\Re_{0}$ relative to the form $B_{\lambda}(x, y)=\lambda([x, y])$. Hence $\Re_{0}$ has codimension two in $\Re$. Let $X^{*} \in \mathcal{N}$ be such that $B_{\lambda}\left(X^{*}, X\right)=1, B_{\lambda}\left(X^{*}, Y\right)=0$. Let $Y^{*}=J X^{*}$ so $B_{\lambda}\left(Y^{*}, Y\right)=1, B_{\lambda}\left(Y^{*}, X\right)=0$.

Let $\mathscr{P}_{1}=\mathscr{P}_{0} \cap\left(\mathscr{T}_{0}\right)_{c}$. Let $\lambda_{0}=\lambda \mid \mathscr{T}_{0}$ and $\mathscr{T}_{00}=\left(\operatorname{ker} \lambda_{0}\right) \cap \mathscr{T}_{0} . \mathscr{T}_{00}$ is an ideal of $\Re_{0}$. We may assume that $X$ and $Y$ span $\Re_{00}$. We shall let " $\sim$ "denote image in $\mathscr{\Re}_{0} / \mathscr{T}_{00}$. Then $\left(\tilde{\mathscr{T}}_{0}, \tilde{\lambda}_{0}, \tilde{\mathscr{P}}_{1}\right)$ is a totally complex triple for $\tilde{\mathscr{T}}_{0}$. The following lemma will be our main induction step in the proof of our theorem.

LEMMA 23. If $q=0, H^{q}\left(\mathscr{P}_{0}, C^{\lambda}\right)=\{0\}$. If $q \geqslant 1$, there is a continuous one to one mapping of $H^{q}\left(\mathscr{P}_{0}, C^{\lambda}\right)$ onto $H^{q-1}\left(\tilde{\mathscr{P}}_{1}, C^{\tilde{\lambda}_{0}}\right)$.

Granted this lemma, our theorem follows, for the signature of the form corresponding to $\tilde{\lambda}_{0}$ is easily seen to be $p-1$. Hence, by induction, $H^{q}\left(\mathscr{P}_{0}, C^{\lambda}\right)=\{0\}$ if $q-1 \neq(n-2)-(p-1) \Leftrightarrow q \neq n-p$. If $q=n-p$, then $q \neq 0$ for $\phi$ must have at least one negative eigenvalue on $\mathfrak{T}_{00} \times \operatorname{span}\left\{X^{*}, Y^{*}\right\}$. Hence, by induction $H^{q}\left(\mathscr{P}_{0}, C^{\lambda}\right)$ is one dimensional and Hausdorff.

Proof (OF Lemma 23). $\mathfrak{T}_{0}$ is contained in a maximal subordinate subalgebra $\mathfrak{R}$ of $\Re$ and $\mathscr{R} \subset \Re_{0}$. Let $\eta$ be the character of $\mathscr{N}$ corresponding to $\lambda(\mathfrak{R}$ is considered as a group here) and let $U^{\eta}$ be the induced representation $U^{\eta}=$ ind(T, $\Re, \eta)$. Let $C^{\eta}=C^{\infty}\left(U^{\eta}\right)$. We take $X \in \operatorname{ker} \lambda$ so $Y$ also belongs to ker $\lambda$. 
Let $\mathfrak{T}_{00}=\mathfrak{K}_{0} \cap \operatorname{ker} \lambda=\operatorname{span}\{X, Y\}$. Let $\mathfrak{T}_{0}^{*}$ denote the set $\operatorname{span}\left\{X^{*}, Y^{*}\right\}$. Let $\mathcal{T}$ be a vector complement to $\mathscr{N}$ in $\mathfrak{K}_{0}$. Then $\mathscr{N}=\mathfrak{R} \cdot \mathcal{T} \cdot \mathfrak{T}_{0}^{*}$ where the multiplication is the group multiplication. This decomposition gives rise to a realization of $U^{\eta}$ in $L^{2}\left(\mathcal{T} \times \mathfrak{T}_{0}^{*}\right) \approx L^{2}(\mathcal{T} \times \mathbf{C})$ where we map $C$ into $\mathfrak{T}_{0}^{*}$ by mapping $x+i y \rightarrow x X^{*}+y Y^{*}$. This realization is obtained by restriction to the set $\mathfrak{T} \cdot \mathfrak{T}_{0}^{*}$. In this realization $C^{\eta}=S(\mathcal{T} \times \mathbf{C})([5])$. Let us compute $U^{\eta}(m)$ for $m=s X+t Y \in \mathfrak{T}_{0}$. Let $t \cdot m_{0} \in \mathcal{T} \cdot \mathscr{T}_{0}^{*}$. Then for $f \in \mathcal{H}\left(U^{\eta}\right)$,

$$
U^{\eta}(m) f\left(t m_{0}\right)=f\left(t m_{0} m\right)=f\left(m^{\prime} t m_{0}\right)=\eta\left(m^{\prime}\right) f\left(t m_{0}\right)
$$

where $m^{\prime}=m_{0} m m_{0}^{-1}$ since $\mathcal{T}$ centralizes $\mathfrak{T}_{0}$. From Campbell-Hausdorff $m^{\prime}=$ $m+\left[m_{0}, m\right]=m+(x s+y t) Z_{0}$ where $\lambda\left(Z_{0}\right)=1, Z_{0} \in \mathscr{Z}_{0}$. From this we see $\partial U^{\eta}(X-i Y) f(t, z)=i z f(t, z)$ for $t \in \mathcal{T}, z \in \mathbf{C}$.

Now let $\mathscr{K}=\operatorname{span} X-i Y$. From the above calculation $\left(C^{\eta}\right)^{\mathscr{K}}=0$ so Hochschild-Serre implies that

$$
H^{q}\left(\mathscr{P}_{0}, C^{\eta}\right) \approx H^{q-1}\left(\mathscr{P}_{0} / \mathcal{K}, H^{1}\left(\mathcal{K}, C^{\eta}\right)\right) .
$$

$H^{1}\left(\mathcal{K}, C^{\eta}\right)$ is isomorphic with $C^{\eta} / \mathscr{K} C^{\eta}$.

Sublemma 24. $f \in \mathcal{K} C^{\eta}$ iff $\partial U\left(X^{*}-i Y^{*}\right)^{n} f(t, 0)=0$ for all $t \in \mathcal{T}$ and all $n \in N$.

Proof. This could be proven directly from Taylor's formula. However, it is easier to use Hörmander [9]. Specifically according to [9], there is a continuous inverse to the mapping $T$ of $\mathcal{S}(\mathcal{T} \times \mathbf{C})$ into $\delta(\mathcal{T} \times \mathbf{C})$ defined by multiplication by $i z$. Hence the image of $\delta(\mathcal{T} \times \mathbf{C})$ under $\mathscr{K}$ is closed. It follows that $f \in \mathcal{K} C^{\eta}$ iff $\langle f, \phi\rangle=0$ for all $\phi$ which annihilate $C^{\eta}$-i.e. for all $\phi$ such that $\phi \circ T=0$. But $\phi \circ T=0$ implies that $\phi$ is supported in $\mathcal{T} \times 0$. Hence $\phi$ is a finite sum of the form

$$
\phi=\sum_{n, m}\left(\frac{\partial}{\partial z}\right)^{m}\left(\frac{\partial}{\partial z}\right)^{n} \phi_{n, m}
$$

where $\phi_{n, m} \in \mathcal{S}^{\prime}(\mathcal{T})$. (We imbed $\mathcal{S}^{\prime}(\mathcal{T})$ in $\mathcal{S}^{\prime}(\mathcal{T} \times \mathbf{C})$ by means of the transpose of the restriction mapping from $\delta(\mathcal{T} \times \mathbf{C})$ to $\delta(\mathcal{T} \times 0)$.) Upon applying $\phi$ to functions of the form $g$ where $g(t, z)=f_{n, m}(z) h(t)$ where $h(t) \in \mathcal{S}(\mathcal{T})$ and $f_{n, m}(z)=$ $z^{m} \bar{z}^{n}$ on a neighborhood of 0 in $C$, we see that $\phi_{n, m}=0$ if $n \neq 0$. Hence, $\phi=\Sigma(\partial / \partial z)^{n} \phi_{n}$. Conversely, any such $\phi$ is orthogonal to $i z \mathcal{S}(\mathcal{T} \times \mathbf{C})$ since $(\partial / \partial z)^{n}(i z f)=i z(\partial / \partial z)^{n} f$. This implies that $f \in \mathcal{K} C^{\eta}$ iff $(\partial / \partial z)^{n} f(t, 0)=0$ for all $n \in N$ and $t \in \mathcal{T}$. Our lemma will follow from the following sublemma.

Sublemma 25. For all $n, \partial U\left(X^{*}-i Y^{*}\right)^{n} f(t, 0)=2^{n}(\partial / \partial z)^{n} f(t, 0)$ for all $t \in \mathcal{T}$ and $f \in \mathcal{S}(\mathcal{T} \times \mathbf{C})$.

Proof. It suffices, by a density argument, to assume that as a function of $x$ and $y, f$ is real analytic. We consider $f$ as a function on $\mathcal{T} \cdot \Re_{0}^{*} \subset \Re$ and we extend $f$ analytically to an open subset of $\mathcal{T} \cdot\left(\mathscr{T}_{0}^{*}\right)_{c}$ in $\Re_{c}$. Then let $W^{*}=X^{*}-i Y^{*}$,

$$
\left(\partial U^{\eta}\left(W^{*}\right)\right)^{n} f(x)=\left.(d / d s)^{n}\right|_{s=0} f\left(x \cdot\left(s W^{*}\right)\right) .
$$


In particular

$$
\begin{aligned}
\partial U^{\eta}\left(W^{*}\right)^{n} f(t, 0) & =\left.(d / d s)^{n}\right|_{s=0} f\left(t, s X^{*}-i s Y^{*}\right) \\
& =2^{n}(\partial / \partial z)^{n} f(t, 0) . \quad \text { Q.E.D. }
\end{aligned}
$$

Using the above sublemma we may easily describe $H^{1}\left(\mathcal{K}, C^{\eta}\right)$. First we describe the dual space $H^{1}\left(\mathcal{K}, C^{\eta}\right)^{*}$. The dual space is the annihilator of $\mathscr{K} C^{\eta}$ in $\left(C^{\eta}\right)^{*}=$ $\mathcal{S}^{\prime}(\mathcal{T} \times \mathbf{C})$. From the above sublemma, this annihilator is isomorphic under the mapping $\phi \otimes P(z) \rightarrow P(2 \partial / \partial z)(\phi \otimes \delta)$ with $\delta^{\prime}(\mathcal{T}) \otimes \mathbf{C}[z]$ where $\mathbf{C}[z]$ is the space of all complex polynomials in the indeterminant $z$. We topologize $\mathbf{C}[z]$ as the direct limit of the spaces of polynomials of degree $n$. These spaces are given their Euclidean topologies. It is easily seen from the closed graph theorem that the isomorphism above is also topological. $H^{1}\left(\mathcal{K}, C^{\eta}\right)$ is reflexive since $\delta(\mathcal{J} \times \mathbf{C})$ is. Hence $H^{1}\left(\mathscr{K}, C^{\eta}\right) \approx\left(\mathcal{S}^{\prime}(\mathcal{T}) \otimes \mathbf{C}[z]\right)^{*}$.

The dual of $\mathbf{C}[z]$ is the space $\mathbf{C}[z]]$ of formal power series where $\left\langle f(z), g^{\prime}(z)\right\rangle=$ $\sum f_{i} g_{i}$ for $f(z) \in \mathbf{C}[z]$ and $g(z) \in \mathbf{C}[[z]]$. Hence $H^{1}\left(\mathcal{K}, C^{\eta}\right) \approx \delta(\mathcal{T}) \otimes \mathbf{C}[[z]]$. Under this isomorphism $\partial U^{n}\left(X^{*}-i Y^{*}\right)$ is mapped onto the mapping which sends $f \otimes z^{n} \rightarrow f \otimes z^{n-1}$ for $n \geqslant 1$ and $f \otimes z^{0} \rightarrow 0$.

Let $\mathcal{K}^{\prime}=\operatorname{span}\left\{X^{*}-i Y^{*}\right\}$. Then from the above comments, $\mathcal{K}^{\prime}$ is surjective on $H^{1}\left(\mathcal{K}, C^{n}\right)$. Hence $H^{1}\left(\mathcal{K}^{1}, H^{1}\left(\mathcal{K}, C^{\eta}\right)\right)$ is zero. The operator $X^{*}-i Y^{*}=K$ has an inverse in $H^{1}\left(\mathcal{K}, C^{\eta}\right)$ given by $f \otimes z^{n} \rightarrow f \otimes z^{n+1}$. If follows from Hochschild-Serre that if $q>1$,

$$
\begin{aligned}
H^{q-1}\left(\mathscr{P}_{0} / \mathscr{K}, H^{1}\left(\mathcal{K}, C^{\eta}\right)\right) & \approx H^{q-1}\left(\mathscr{P}_{0} /\left(\mathscr{K}+\mathscr{K}^{\prime}\right), H^{1}\left(\mathcal{K}, C^{\eta}\right)^{\mathscr{K}^{\prime}}\right) \\
& =H^{q-1}\left(\mathscr{P}_{0} /\left(\mathcal{K}+\mathcal{K}^{\prime}\right), \mathcal{S}(\mathcal{T})\right)
\end{aligned}
$$

since the kernel of $X^{*}-i Y^{*}$ in $H^{1}\left(\mathscr{K}, C^{\eta}\right)$ is $z^{0} \otimes S(\mathscr{T})$. From Lemma 18 , the isomorphism is a topological isomorphism. If $q=1$, the above equality is trivial since

$$
\begin{aligned}
H^{0}\left(\mathscr{P}_{0} / \mathscr{K}, H^{1}\left(\mathscr{K}, C^{\eta}\right)\right) & =\bigcap_{W \in \mathscr{P}_{0} / \mathscr{K}} \operatorname{ker}\left(\partial U^{\eta}(W) \text { in } H^{1}\left(\mathscr{K}, C^{\eta}\right)\right) \\
& =\bigcap_{W \in \mathscr{P}_{0} /\left(\mathscr{K}+\mathcal{K}^{\prime}\right)}\left(\operatorname{ker} \partial U^{\eta}(\mathscr{K}) \text { in } \operatorname{ker} X^{*}-i Y^{*}\right) .
\end{aligned}
$$

Since $C^{\infty}\left(U^{\eta}\right)$ is identified with $\delta(\mathcal{T})$, it can easily be seen that

$$
H^{q-1}\left(\mathscr{P}_{0} /\left(\mathscr{K}+\mathscr{K}^{\prime}\right), H^{1}\left(\mathcal{K}, C^{\eta}\right)\right) \approx H^{q-1}\left(\mathscr{P}_{1}, C^{\eta}\right) \text { Q.E.D. }
$$

This finishes the proof of Theorem 16.

III. Applications to the study of automorphic forms on a nilmanifold. Now, let $(\mathscr{T}, \mathcal{P}, \lambda)$ be as in $\S I$. Suppose in addition there is a discrete subgroup $\Gamma \subset N$ such that $\Gamma \cap Z \subset \operatorname{ker} \chi$ where $\chi$ is as in $\S I$, i.e. $\chi(z)=\exp i \lambda\left(\log _{N}(z)\right)$. As in $\S I$ we set $\Delta=\Gamma Z, M=\Delta \backslash N$. We define the bundle $F$ as in $\S I$. We are interested in knowing when $\mathcal{F}^{p}(F)$ is nonzero. The following theorem is now an easy consequence of the results above. 
THEOREM 26. $\mathcal{H}^{p}(F)$ is nonzero iff $p=$ signature $\phi$ where $\phi$ is the form on $\mathscr{P} \times \mathscr{P}$ defined by $\phi(x, y)=-i \lambda([x, \bar{y}])$. Furthermore, for this valve of $p$, the dimension of $\mathcal{H}^{p}(F)$ is the multiplicity of $U^{\lambda}$ in the quasi-regular representation of $N$ in $L^{2}(\Gamma \backslash N)$. ( $U^{\lambda}$ is the representation corresponding to $\lambda$ under the Kirillov map.)

Proof. From the compactness of $M, \mathcal{F}^{p}(F)$ is isomorphic with $H^{p}(F)$, which in turn is isomorphic with $H^{p}\left(\mathscr{P}_{0}, C^{\mu}\right)$ (see the beginning of $\left.\S I\right)$. From Lemma $11, U^{\mu}$ is primary and quasi-equivalent with $U^{\lambda}$. From the compactness of $M, U^{\mu} \approx n$. $U^{\lambda}$ where $n$ is the (finite) multiplicity of $U^{\lambda}$ in $U^{\mu}$. Hence $C^{\mu}=n \cdot C^{\lambda}$ and $H^{p}\left(\mathscr{P}_{0}, C^{\mu}\right)=n H^{p}\left(\mathscr{P}_{0}, C^{\lambda}\right)$. By Theorem $16, H^{p}\left(\mathscr{P}_{0}, C^{\lambda}\right)$ is either one dimensional or zero dimensional, depending upon whether $p$ equals the signature of $\phi$ or not. Q.E.D.

Next we prove the theorem on "holomorphic difference" equations mentioned in the introduction. Let $U$ be an open subset of $M$. An element $f$ of $\Gamma^{\infty}(U, F)$ is said to be holomorphic if $\nabla_{X} f=0$ for all $X \in \mathcal{P}$. The set of germs of holomorphic sections of $F$ forms a sheaf $\Omega(F)$. We use this sheaf to form the holomorphic cohomology groups $H^{p}(M, \Omega(F))$. It follows from the Dolbeault theorem that $H^{p}(M, \Omega(F))$ is isomorphic with $H^{p}(F)$, which we just computed. We shall relate $H^{P}(M, \Omega(F))$ to a group-theoretic cohomology space.

Specifically, let $E$ be the $Z \backslash N$ bundle of $\S$. Let $\Gamma\left(N^{\prime}, \Omega(E)\right)$ be the space of global holomorphic sections of $E$ over $N^{\prime}=Z \backslash N$. Since $Z$ is central, $N$ acts on $E$ according to the formula $g[(x, c)]=[(g x, c)]$ where $g, x \in N$ and $c \in \mathbf{C}$. Since $\Gamma \cap Z \subset \operatorname{ker} \chi, \Gamma \cap Z$ acts trivially so $\Gamma^{\prime}=\Gamma / \Gamma \cap Z$ acts on $E$. This action preserves holomorphicity in $\Omega(E)$.

Let $C^{p}(\Gamma, \Omega(E))$ be the set of mappings $f$ of $\Gamma^{p}$ into $\Gamma\left(N^{\prime}, \Omega(E)\right)$ such that $f\left(\alpha_{1}, \ldots, \alpha_{p}\right)=0$ whenever any $\alpha_{i}=e$. These are the normalized cochains. We define the nonhomogeneous coboundary operators

$$
d: C^{p}\left(\Gamma^{\prime}, \Omega(E)\right) \rightarrow C^{p+1}\left(\Gamma^{\prime}, \Omega(E)\right)
$$

by

$$
\begin{aligned}
d f\left(\alpha_{1}, \ldots, \alpha_{p+1}\right)= & \alpha_{1} \cdot f\left(\alpha_{2}, \ldots, \alpha_{p+1}\right) \\
& +\sum_{i=1}^{n}(-1)^{i} f\left(\alpha_{1}, \ldots, \alpha_{i} \alpha_{i+1}, \ldots, \alpha_{n+1}\right) \\
& +(-1)^{n+1} f\left(\alpha_{1}, \ldots, \alpha_{p}\right) .
\end{aligned}
$$

The operator $d$ is just the usual group-theoretic coboundary operator.

THEOREM 27. For all $p \geqslant 0, H^{p}\left(\Gamma^{\prime}, \Omega(E)\right)$ and $H^{p}(M, \Omega(F))$ are isomorphic.

Proof. Let us recall first the definition of $H^{P}(M, \Omega(F))$. Let $Q$ be a finite open covering of $M$ such that each $U \in \mathcal{U}$ is diffeomorphic with an open ball in $\mathbf{C}^{n}$ under a complex analytic map. We shall fix $\mathcal{O}$ throughout this discussion and shall not carry $\mathcal{U}$ in our notation. Let $I$ be an index set for $\mathcal{U}$ and let $I_{p}=$ $\left\{\left(i_{1}, \ldots, i_{p}\right) \mid i_{j} \in I\right.$ and $\left.U_{i_{1}} \cap \cdots \cap U_{i_{p}} \neq \varnothing\right\}$. For $i \in I_{p}$, let $U_{i}=U_{i_{1}}$ $\cap \cdots \cap U_{i_{p}}$. Let $C^{p}(M, \Omega(F))$ be the set of mappings $f$ of $I_{p+1}$ into sections of $F$ such that $f(i) \in \Gamma\left(U_{i}, \Omega(F)\right)$ and $f\left(i_{1}, \ldots, i_{p+1}\right)=\operatorname{sgn} \sigma f\left(i_{\sigma(1)}, \ldots, i_{\sigma(p+1)}\right)$ for all 
permutations $\sigma$ in $\pi_{p+1}$. The boundary operator $d: C^{p}(M, \Omega(F)) \rightarrow C^{p+1}(M, \Omega(F))$ is defined by

$$
d f\left(i_{1}, \ldots, i_{p+2}\right)=\sum(-1)^{i} f\left(i_{1}, \ldots, i_{j}, \ldots, i_{p+2}\right) \mid U_{\left(i_{1}, \ldots, i_{p+2}\right)} .
$$

The corresponding cohomology groups do not depend on $\mathcal{Q}$. They are isomorphic with $H^{p}(M, \Omega(F))$.

We shall prove our isomorphism theorem by defining a double complex $C^{p, q}$ that interpolates between the spaces $C^{p}\left(\Gamma^{\prime}, \Omega(E)\right)$ and $C^{p}(M, \Omega(F))$.

Let $\rho: N^{\prime} \rightarrow M$ be the usual projection mapping and let $\rho^{-1}(U)$ be the open covering of $N^{\prime}$ gotten from pulling elements of $\mathcal{U}$ back by $\rho$. We may assume that $\mathcal{U}$ has been chosen so that the restriction of $\rho$ to any connected component of $\rho^{-1}(U)$ is an analytic diffeomorphism for any $U \in \mathcal{Q} . \rho^{-1}(\mathcal{Q})$ is indexed by the same set $I$ as $\mathscr{Q}$ is. We define $C^{p}\left(N^{\prime}, \Omega(E)\right)$ relative to $p^{-1}(\mathcal{Q})$ just as $C^{p}(M, \Omega(F))$ was defined relative to $\mathcal{U}$ and we define the boundary operator

$$
d: C^{p}\left(N^{\prime}, \Omega(E)\right) \rightarrow C^{p+1}\left(N^{\prime}, \Omega(E)\right)
$$

just as before. Let $C^{p, q}$ be the set of mappings $f$ of $\Gamma^{q}$ into $C^{p}\left(N^{\prime}, \Omega(E)\right)$ which are normalized as before by saying that $f$ is zero whenever any one of its $\Gamma$ arguments is zero. (If $q=0, \Gamma^{q}$ is defined to a singleton set $\{0\}$ so $C^{p, 0}=C^{p}\left(N^{\prime}, \Omega(F)\right.$ ).) The $C^{p}\left(N^{\prime}, \Omega(E)\right)$ boundary operator defines a boundary operator $d^{\prime \prime}: C^{p, q} \rightarrow C^{p+1, q}$. We also define a boundary operator $d^{\prime}: C^{p, q} \rightarrow C^{p, q+1}$ by means of the formula for the $C^{q}\left(\Gamma^{\prime}, \Omega(F)\right)$ boundary.

There is an obvious mapping $d_{q}^{\prime}$ of $C^{q}\left(\Gamma^{\prime}, \Omega(F)\right)$ into $C^{0, q}$ defined by restriction of sections of $\Omega(F)$ to $\rho^{-1}(\mathcal{Q})$. There is also a mapping $d^{\prime \prime}$ of $C^{p}(M, \Omega(F)$ ) into $C^{p, 0}=C^{p}\left(N^{\prime}, \Omega(E)\right)$ defined as follows. The mapping $\rho$ gives rise to a bundle map $\rho^{*}: E \rightarrow F$ given by $\rho^{*}[(z x, c)]=[(\Delta x, c)]$.

Given any section $f$ of $F$ over some open subset $U$ of $M$, there is a unique section $\tilde{\rho} f$ over $\rho^{-1}(U)$ such that $\rho^{*}(\tilde{\rho} f)=f \circ \rho$. The mapping $\tilde{\rho}$ gives rise to the mapping $d^{\prime \prime}$ in the obvious way.

Before summarizing what we have, we make one more observation. The space $H^{0}\left(\Gamma^{\prime}, \Omega(E)\right)$ is the space of $\Gamma^{\prime}$ invariant holomorphic sections of $E$. The space $H^{0}(M, \Omega(F))$ is the space of global holomorphic sections of $F$ over $M$. $\tilde{\rho}$ establishes an isomorphism between these spaces. Let $H^{0}\left(\Gamma^{\prime}, \Omega(F)\right)=H=H^{0}(M, \Omega(F))$. Then we have injections $\varepsilon_{1}: H \rightarrow C^{0}\left(\Gamma^{\prime}, \Omega(E)\right), \varepsilon_{2}: H \rightarrow C^{0}(M, \Omega(F))$. The complexes $\left(H, \varepsilon_{1}, C^{p}\left(\Gamma^{\prime}, \Omega(F)\right)\right)$ and $\left(H, \varepsilon_{2}, C^{p}(M, \Omega(E))\right)$ are augmented chain complexes. It follows from Theorem 3.1, Chapter 6 of [7] that $H^{p}(M, \Omega(F)) \approx H^{p, 0}$, $H^{q}\left(\Gamma^{\prime}, \Omega(E)\right) \approx H^{0, q}$. Where $H^{i j}$ denotes the $i, j$ cohomology group of the double complex. To finish our theorem we shall show that $H^{i, j} \approx H^{p, q}$ whenever $i+j=p$ $+q$. This will imply that $H^{p, 0} \approx H^{0, p}$, as desired. From Theorem 3.2 of [7], it suffices to show

LEMMA 28. In the $C^{p, q}$ complex, the $d^{\prime}$ and the $d^{\prime \prime}$ sequences are exact at $C^{p, q}$ for all $p$ and $q$.

Proof. We consider first the $d^{\prime \prime}$ sequence. It suffices to show exactness at $C^{p, 0}$ since $C^{p, q}$ is just the space of maps of $\Gamma^{q}$ into $C^{p, 0}$ which satisfy the normalization 
condition and, as is well known, normalization does not affect cohomology. For $p=0$ there is nothing to show as the complex begins at $(0,0) . d^{\prime \prime}$ will be exact at $C^{p, 0}$ iff the cohomology of the complex $\left(d^{\prime \prime}, C^{p, 0}\right)$ is zero for $p>0$. This cohomology is isomorphic with $H^{p}\left(N^{\prime}, \Omega(E)\right)$ by the choice of $\mathscr{Q}$. But $E$ is holomorphically trivial. To see this let $p_{1}=\exp _{N_{c}} \odot$ and $P_{2}=\exp _{N_{c}} \overline{\mathcal{P}}$. Let $\chi_{i}$ be the character of $P_{i}$ gotten by restricting exp $i \lambda_{0} \log _{N_{c}}$ to $P_{i}$. It is easily seen that $N_{c}=P_{2} P_{1}$. Let $\psi$ be the function on $N_{c}$ defined by $\psi\left(p_{2} p_{1}\right)=\chi_{2}\left(p_{2}\right) \chi_{1}\left(p_{1}\right) . \psi$ is well defined and the restriction of $\psi$ to $N$ defines a global holomorphic section of $E$ which is nowhere zero. Hence $\psi$ defines a holomorphic trivialization of $E$. It follows that $H^{p}\left(N^{\prime}, \Omega(E)\right)=0$ if $p>0$.

Next we consider the $d^{\prime}$ sequence. Again, we claim that it suffices to show exactness at $C^{0, q}$ for $q>0$. To see this suppose that exactness has already been shown at $C^{0, q}$ for all $q$ and for all $\mathscr{U}$ satisfying the above assumptions. Let $\mathcal{U}_{p}$ be the covering of $M$ defined by $\mho_{p}=\left\{U_{i} \mid i \in I_{p}\right\}$. $\mathscr{U}_{p}$ satisfies the same assumptions as $\mathcal{Q}$ and $C_{\vartheta}^{p, q} \subset C_{\vartheta_{p}}^{0, q}$ where the subscript indicates the covering used to define the space. The $d^{\prime}$ operator on $C_{Q}^{p, q}$ is the restriction of the $d^{\prime}$ operator of $C_{Q_{p}^{0}}^{0, q}$. Let $f$ be a $d^{\prime}$-cocycle of $C_{Q_{i}^{p}}^{p, q}$. Then from exactness of $C_{\hat{Q}_{p}}^{0, q}$, there is a $d^{\prime}$-cochain $g$ of $C_{Q_{p}}^{0, q}$ such that $d^{\prime} g=f$. We may assume that as a mapping of $I_{p}$ onto $\mathscr{U}_{p}, g$ is antisymmetric since antisymmetrization commutes with $d^{\prime}$ and leaves $f$ invariant. Hence $f$ is a $d^{\prime}$ boundary in $C_{Q}^{p, q}$. This shows exactness at $C^{p, q}$.

To prove exactness at $C^{0, q}$, let $f$ be a cocycle of $C^{0, q}$. Let us fix $i \in I$ and set $U=U_{i}$. Let $V$ be any connected component of $\rho^{-1}(U)$. By assumption $\rho \mid V$ is a diffeomorphism onto $U$ and $\rho^{-1}(U)=\Gamma^{\prime} V$. Furthermore $\gamma V \cap V \neq \varnothing$ for $\gamma \in \Gamma^{\prime}$ iff $\gamma=e$. Let $M$ be the mapping of $\Gamma^{q-1}$ into $\Gamma\left(\rho^{-1}(U), \Omega(E)\right)$ defined by

(1) $h\left(\gamma_{1}, \ldots, \gamma_{q-1}\right) \mid V=0$,

(2) $h\left(\gamma_{1}, \ldots, \gamma_{q-1}\right)\left|\gamma V=\gamma^{-1} f(i)\left(\gamma, \gamma_{1}, \ldots, \gamma_{q-1}\right)\right| \gamma V$.

Sublemma 29. $d h\left(\gamma_{1}, \ldots, \gamma_{q}\right)=f(i)\left(\gamma_{1}, \ldots, \gamma_{q}\right)$.

Proof. We begin by considering this equality on $V$.

$$
\begin{aligned}
d h\left(\gamma_{1}, \ldots, \gamma_{q}\right)= & \gamma_{1} h\left(\gamma_{2}, \ldots, \gamma_{q}\right) \\
& -\sum_{i} h\left(\gamma_{1}, \ldots, \gamma_{i} \gamma_{i+1}, \ldots, \gamma_{q}\right)(-1)^{i}+h\left(\gamma_{1}, \ldots, \gamma_{q-1}\right) \\
= & \gamma_{1} h\left(\gamma_{2}, \ldots, \gamma_{q}\right)
\end{aligned}
$$

on $V$. By definition

$$
\begin{aligned}
\gamma_{1} h\left(\gamma_{2}, \ldots, \gamma_{q}\right) \mid V & =\gamma_{1}\left(\gamma_{1}^{-1} f(i)\left(\gamma_{1}, \ldots, \gamma_{q}\right)\right) \mid V \\
& =f(i)\left(\gamma_{1}, \ldots, \gamma_{q}\right) \mid V
\end{aligned}
$$

so our claim is true on $V$ at least. Let $d h=k$. Then $d k=0$ so

$$
\begin{aligned}
\gamma_{1} k\left(\gamma_{2}, \ldots, \gamma_{q+1}\right)= & -\sum(-1)^{i} k\left(\gamma_{1}, \ldots, \gamma_{i} \gamma_{i+1}, \ldots, \gamma_{q+1}\right) \\
& -(-1)^{n+1} k\left(\gamma_{1}, \ldots, \gamma_{q}\right) .
\end{aligned}
$$

Since $d f(i)=0$ and $f(i)=k$ on $V$, we conclude that

$$
\gamma_{1} k\left(\gamma_{2}, \ldots, \gamma_{q+1}\right)=\gamma_{1} f\left(\gamma_{2}, \ldots, \gamma_{q+1}\right)
$$


on $V$ for all $\gamma_{i} \in \Gamma^{\prime}$. But this implies that $f(i)=d h$ on $\Gamma^{\prime} V=\rho^{-1}(U)$. This proves the sublemma. Q.E.D.

If we now allow $i$ to vary and let $h(i)$ be the corresponding $h$ to $f(i)$ on $\rho^{-1}\left(U_{i}\right)$, then it is clear that $d^{\prime} h=f$. This proves the required exactness and proves our theorem. Q.E.D.

Corollary 30. $H^{p}\left(\Gamma^{\prime}, \Omega(E)\right)$ is zero unless $p$ equals the signature of $\phi$ in which case $H^{p}\left(\Gamma^{\prime}, \Omega(E)\right)$ is one dimensional.

IV. Examples. We begin with a general construction of a class of groups possessing the structure hypothesised in the previous sections. Our class is motivated by Howe [11]. It has also been studied in [17] and [18].

Let $\mathcal{Q}$ be a nilpotent, finite dimensional, abelian, associative algebra (without unit of course). Suppose $\mathbb{Q}$ possesses a nondegenerate, bilinear, symmetric form $B$ which satisfies $B(x y, w)=B(x, y w)$ for all $x, y, w \in \mathbb{Q}$. We make the set $\mathfrak{V}=\mathbb{Q}$ $\times \mathscr{Q}$ into an algebra by setting $\left(x_{1}, x_{2}\right)\left(y_{1}, y_{2}\right)=\left(x_{1} y_{1}, x_{1} y_{2}\right)$. Let $\alpha$ be the bilinear form on $\mathfrak{V} \times \mathfrak{V}$ defined by $\alpha(x, y)=B\left(x_{1}, y_{2}\right)$. Then $\alpha$ satisfies $\alpha(x y, w)=$ $\alpha(x, y w)=\alpha(y x, w)$. We use $\alpha$ to define an algebra structure on $\Re=\mathcal{T} \times \mathbf{R}$ by setting $(x, t)(y, s)=(x y, \alpha(x, y))$ for $x, y \in \mathcal{V}$ and $s, t \in \mathbf{R}$. We make $\mathscr{T}$ into a Lie algebra by setting $[u, v]=u v-v u$ in $\Re$. Let $\lambda \in \Re^{*}$ be the functional which maps $(v, t) \rightarrow \lambda_{0} t$ for some real number $\lambda_{0}$. The complexification of $\Re$ is $\varkappa_{c}=\mathscr{V}_{c}$ $\times \mathbf{C}$, where $\mathfrak{V}_{c}=\mathbb{Q}_{c} \times \mathbb{Q}_{c}$. Let $\mathscr{P}_{0} \subset \mathfrak{V}_{c}$ be $\mathscr{P}_{0}=\left\{(x, i x) \mid x \in \mathbb{Q}_{c}\right\}$. Then $\mathscr{P}_{0}$ is a subalgebra of $\mathcal{V}_{c}$ and $\mathscr{P}_{0} \times \mathbf{C}=\mathscr{P}$ is a maximal subordinate, abelian subalgebra of $\Re$ with respect to $\lambda$. The triple $(\Re, \mathcal{P}, \lambda)$ satisfies all of the assumptions of the previous sections.

The Lie group corresponding to $\Re$ is the group $N$ which equals $\Re$ as a set and carries the group law "." defined by $x \cdot y=x+y+x y$. The inverse is $x^{-1}=-x$ $+x^{2}-x^{3}+\cdots$. The exponential map is

$$
\exp _{\Re} x=x+x^{2} / 2 !+\cdots+x^{n} / n !+\cdots \cdot
$$

Now suppose there is a vector lattice $\Lambda \subset \mathbb{Q}$ such that $\Lambda \cdot \Lambda \subset \Lambda$ and $\alpha$ : $\Lambda \times \Lambda \rightarrow \mathbf{Z}$. Then the set $\Gamma=(\Lambda \times \Lambda) \times \mathbf{Z}$ is a discrete, cocompact subgroup of $\Re$.

Before discussing specific instances of this construction, we shall construct a holomorphic trivialization of the bundle $E$. We could (and perhaps should) use the canonical trivialization defined in the proof of Theorem 27 above. However, this trivialization is not as convenient computationally as the one we shall now define. Let $\mathfrak{T} \subset \Re$ be the subalgebra (subgroup) $(0 \times \mathcal{Q}) \times \mathbf{R}$. Let $\chi_{0}$ be the character of $\mathscr{N}$ defined by $\chi_{0}(a, t)=\exp i \lambda_{0} t$ and let $\chi$ be the character of $\mathscr{P}$ defined by $\chi(p, t)=\exp i \lambda\left(\log _{N}(p, t)\right)$ for $(p, t) \in \mathscr{P}_{0} \times \mathbf{R}$. Then $\mathfrak{R} * \mathscr{P}=\mathfrak{T}_{c}$ so we may define a function $w$ on $N$ by $w(m * p)=\chi_{0}(m) \chi(p) . w$ defines a nowhere zero holomorphic section of $F$ which gives rise to a holomorphic trivialization of $F$. This function is computed in $\$ 3$ of [17] where it is shown that

$$
w((x, y), t)=\exp \left(-\lambda_{0} 1(x)+\lambda_{0} i t\right)
$$


where $1(x)=\sum_{n=2}^{\infty}(-1)^{n} B\left(x, x^{n-1}\right) / n$. The complex structure on $\mathbb{Q} \times \mathbb{Q}$ is defined by the map $J$ where $J(a, b)=(-b, a)$. So $F$ is isomorphic with $\mathbb{Q}_{c} \times \mathbf{C}$. The holomorphic sections are just holomorphic functions on $\mathbb{Q}_{c}$. The subgroup $\Gamma^{\prime}=\Gamma$ $\cap Z \backslash \Gamma$ is $\Lambda_{c} \subset \mathbb{Q}_{c}$ and in terms of the trivialization, the $\Gamma^{\prime}$ action on $f \in$ $\Gamma\left(N^{\prime}, \Omega(F)\right)$ is $\gamma^{-1} f(z)=w(\gamma * z) w(z)^{-1} f(\gamma * z)$ for $\gamma \in \Lambda_{c}, z \in \mathbb{Q}_{c} \subset \mathfrak{V}$. This computes to be

$$
\gamma^{-1} f(x, y)=f(\gamma *(x, y)) \exp -\lambda_{0}\left(1\left(\gamma_{1}\right)+B\left(\gamma_{1}, x+i y\right)\right) .
$$

(Here we used the identity $1(x+y+x y)=1(x)+1(y)-B(x, y)$ proved in [18].) Now we consider some specific examples.

EXAMPLE I. Let $Q$ be $\mathbf{R}^{n}$ with trivial multiplication. Let $B(x, y)=x \cdot y$ where "." denotes the usual scalar product. Then $\mathfrak{R}$ is just the usual $n$-dimensional Heisenberg group. $\mathscr{T}_{c}$ is $\left(\mathbf{C}^{n} \times \mathbf{C}^{n}\right) \times \mathbf{C}$ and $\mathscr{P}_{0}=\left\{(x, i x) \mid x \in \mathbf{C}^{n}\right\}$. The form $\phi$ is defined by

$$
\begin{aligned}
\phi((x, i x),(y, i y)) & =i \lambda([(x, i x),(\bar{y},-i \bar{y})]) \\
& =i(\alpha(x,-i \bar{y})-\alpha(i x, \bar{y}))=2(x, y)
\end{aligned}
$$

where $(x, y)$ denotes the usual Hermitian scalar product on $\mathbf{C}^{n}$. Hence $\phi$ is positive definite and the signature is $n$. Let $\Lambda=Z^{n}$ be considered as a subset of $Q=\mathbf{R}^{n}$. Form $\Gamma$ as above. Hence $H^{0}\left(\Gamma^{\prime}, F\right)$ is nonzero and $H^{p}\left(\Gamma^{\prime}, F\right)$ is zero for all $p>0$. (See §III for notation.)

The meaning of $H^{0}\left(\Gamma^{\prime}, \Omega(F)\right) \neq 0$ is clear. $F$ has $\Gamma$ invariant global holomorphic sections so there are holomorphic sections of $E$ over $M=\Gamma \backslash N$. These sections are the theta functions of Auslander-Tolimieri. To understand the meaning of $H^{p}\left(\Gamma^{\prime}, \Omega(F)\right)=0$ for $p>0$. Let us consider the $p=1, n=1$ case. $\Gamma^{\prime}=\Gamma / \Gamma \cap Z$ $=\mathbf{Z} \times \mathbf{Z} . \Gamma^{\prime}$ is generated by the elements $\gamma_{1}=(1,0)$ and $\gamma_{2}=(0,1)$. Let $f$ be a cocycle of $C^{1}\left(\Gamma^{\prime}, \Omega(F)\right)$. Let $f_{1}=f\left(\gamma_{1}\right)$ and $f_{2}=f\left(\gamma_{2}\right), f_{i} \in \Gamma\left(N^{\prime}, \Omega(F)\right)$. The fact that $f$ is a cocycle implies in particular $f\left(\gamma_{1} \gamma_{2}\right)=\gamma_{1} f\left(\gamma_{2}\right)+f\left(\gamma_{1}\right), f\left(\gamma_{2} \gamma_{1}\right)=\gamma_{2} f\left(\gamma_{1}\right)$ $+f\left(\gamma_{2}\right)$. Using the abelianness of $\Gamma^{\prime}$ we see $\gamma_{1} f_{2}-f_{2}=\gamma_{2} f_{1}-f_{1}$.

LEMMA 31. Suppose $f_{1}$ and $f_{2}$ are elements of $\Gamma\left(N^{\prime}, \Omega(F)\right)$ such that $\gamma_{1} f_{2}-f_{2}=$ $\gamma_{2} f_{1}-f_{1}$. Then there is a unique cocycle $f$ of $C^{1}\left(\Gamma^{\prime}, \Omega(F)\right)$ such that $f_{i}=f\left(\gamma_{i}\right)$ for $i=1,2$.

Proof. We define $f\left(\gamma_{i}^{n}\right)$ for $n>0$ inductively by

(a) $f\left(\gamma_{i}\right)=f_{i}$,

(b) $f\left(\gamma_{i}^{n}\right)=\gamma_{i} f\left(\gamma_{i}^{n-1}\right)+f\left(\gamma_{i}\right)$.

We define $f\left(\gamma_{i}^{0}\right)=0$ and $f\left(\gamma_{i}^{-n}\right)=-\left(\gamma_{i}^{-n}\right) f\left(\gamma_{i}^{n}\right)$. We then set $f\left(\gamma_{i}^{n} \gamma_{2}^{m}\right)=\gamma_{1}^{n} f\left(\gamma_{2}^{m}\right)+$ $f\left(\gamma_{1}^{m}\right)$. It is easily seen by induction that $f\left(\gamma_{i}^{n+m}\right)=\gamma_{i}^{n} f\left(\gamma_{i}^{m}\right)+f\left(\gamma_{i}^{n}\right)$ for all $n \in Z$. It then follows that $f\left(\gamma_{1}^{n} \gamma_{2}^{m}\right)=\gamma_{2}^{m} f\left(\gamma_{1}^{n}\right)+f\left(\gamma_{2}^{m}\right)$. (One first assumes $n=1$ and works by induction on $m$ and then by induction on $n$.) From this equality it is not hard to show that $f$ is a cocycle. We shall omit the details. Q.E.D.

CoRollary 32. If $f_{1}$ and $f_{2} \in \Gamma\left(N^{\prime}, \Omega(F)\right)$ and $\gamma_{1} f_{2}-f_{2}=\gamma_{2} f_{1}-f_{1}$, then there is a section $g \in \Gamma\left(N^{\prime}, \Omega(F)\right)$ such that $f_{1}=\gamma_{1} g-g$ and $f_{2}=\gamma_{2} g-g$. 
To make this corollary more specific, we holomorphically trivialize the bundle $F$ as above. Our corollary is

COROLlary 33. Suppose $f_{1}$ and $f_{2}$ are entire functions of $z$ and suppose

$$
f_{1}(z+i)-f_{1}(z)=e^{-\lambda_{0}(z+1 / 2)} f_{2}(z+1)-f_{2}(z)
$$

where $\lambda_{0} \in 2 \pi \mathbf{Z}$. Then there is an entire function $g$ such that

$$
f_{1}(z)=e^{-\lambda_{0}(z+1 / 2)} g(z+1)-g(z), \quad f_{2}(z)=g(z+i)-g(z) .
$$

EXAmple II. Let $Q$ be the algebra on one generator $e$ subject to the relation $e^{3}=0$. Let $B$ be the form on $\mathbb{Q} \times \mathbb{Q}$ such that

$$
\begin{aligned}
B\left(e^{i}, e^{j}\right)=0 & \text { if } i+j \neq 3 \\
=1 & \text { if } i+j=3 .
\end{aligned}
$$

Then $B$ satisfies $B(x y, w)=B(x, y w)$ so we may construct $\Re$ as above. Let $\Lambda$ be the span over $Z$ of the elements $\left\{e, e^{2}\right\}$ in $Q$ and let $\Gamma$ be as above. Let $\lambda_{0}=2 \pi$. In this example we shall explicitly describe an automorphic cohomology class for the bundle $F$ over $M=\Delta \backslash N$. First of all, it is easily computed that the signature of the form $\phi$ is +1 so the nonzero cohomology occurs in dimension $2-1=1$. Let $\mu$ be as in $\S I I I$ ( $\mu$ is the trivial extension to $\Delta$ of the character $\chi$ of $Z$ defined by $\left.\chi(((0,0), t))=\exp i \lambda_{0} t\right)$. Let $U^{\mu}$ be the representation $\operatorname{ind}(\Delta, N, \mu)$. Then, from $\S I I$, $H^{1}\left(\mathscr{P}_{0}, C^{\mu}\right) \approx H^{1}(M, F)$. It is easily seen from results of [20] (or especially $\S I I I$ of [18]) that $U^{\mu}$ is irreducible so $U^{\mu}$ is equivalent with $U^{\lambda}$ and $H^{1}\left(\mathscr{P}_{0}, C^{\mu}\right) \approx$ $H^{1}\left(\mathscr{P}_{0}, C^{\lambda}\right)$. We shall construct a basis of $H^{1}\left(\mathscr{P}_{0}, C^{\lambda}\right)$ (which is one dimensional).

Let

$$
\begin{aligned}
\mathscr{K} & =\mathscr{N}^{2}=\operatorname{span}_{R}\{x y \mid x, y \in \mathscr{N}\} \\
& =\underset{R}{\operatorname{span}}\left\{\left(\left(e^{2}, 0\right), 0\right),\left(\left(0, e^{2}\right), 0\right),((0,0), 1)\right\} .
\end{aligned}
$$

Obviously $\mathcal{K}$ is a two sided abelian ideal of $\mathscr{N}$ which is maximal subordinate relative to $\lambda$. Let $K$ denote $\mathscr{K}$ considered as a subgroup. The corresponding character $\chi_{0}$ of $K$ is $\left.\chi_{0}: \dot{(}(a, b), t\right) \rightarrow \exp 2 \pi i t$. The representation $U^{0}=$ ind $\left(K, N, X_{0}\right)$ is equivalent with $U^{\lambda}$. Let $T=\operatorname{span}_{\mathrm{R}}\{((e, 0), 0),((0, e), 0)\} . T$ is not a subgroup of $N$, but $N=K T$. We identify $T$ with $\mathrm{C}$ by mapping $x+i y \rightarrow x e_{1}+$ $y e_{2}$ where $e_{1}=((e, 0), 0)$ and $e_{2}=((0, e), 0)$. We realize $U^{0}$ in $L^{2}(C)$ by restriction of functions in $\mathcal{H}\left(U^{9}\right)$ to $T$. It is easily computed that for $k=\left(\left(a e^{2}, b e^{2}\right), 0\right) \in K$, $\partial U^{0}(k) f(z)=2 \pi i(x b-a y) f(z)$. In particular $E_{1}=\left(\left(e^{2},-i e^{2}\right), 0\right) \in \mathscr{P}_{0}$ and $\partial U^{0}\left(\left(e^{2},-i e^{2}\right), 0\right) f(z)=2 \pi \bar{z} f(z)$. As in the proof of Lemma $23, H^{1}\left(\mathscr{P}_{0}, C^{\lambda}\right) \sim$ $H^{0}\left(\mathscr{P}_{0} / \mathscr{K}_{0}, H^{1}\left(\mathscr{K}_{0}, C^{0}\right)\right.$ where $\mathscr{K}_{0}=\operatorname{span}_{\mathrm{C}} E_{0}$ and $C^{0}=\mathcal{S}(C)$. This isomorphism is obtained by restricting forms in $C^{1}\left(\mathscr{P}_{0}, C^{9}\right)$ to $\mathcal{K}_{0}$. As in the proof of Lemma 23, $H^{1}\left(\mathcal{K}_{0}, C^{9}\right)$ is isomorphic with the space of formal power series over $\mathbf{C}, \mathbf{C}[[z]]$. The isomorphism here is obtained by mapping a function $f \in C^{1}\left(\mathcal{K}, C^{9}\right)$ into $\sum_{n=0}^{\infty}\left(W^{n} f(0)\right) z^{n}$ where $W=\partial U^{0}((e,-i e), 0)$.

Under this isomorphism $W$ maps $Z^{n} \rightarrow Z^{n-1}$ for $n>0$ and $W\left(Z^{0}\right)=0$ so $H^{0}\left(\mathscr{P}_{0} / \mathscr{K}_{0}, H^{1}\left(\mathscr{K}_{0}, C^{9}\right)\right)$ is the space of constant power series. Hence any element 
$f$ of $\delta(\mathbf{C})$ which is nonzero at zero and satisfies $W^{n} f(0)=0$ (e.g. $-f$ is constant in a neighborhood of zero) will correspond to a nonzero element of $H^{0}\left(\mathscr{P}_{0} / \mathcal{K}_{0}, H^{1}\left(\mathscr{K}_{0}, C^{9}\right)\right)$. Let $g$ be the element of $C^{1}\left(\mathscr{P}_{0}, C^{9}\right)$ defined by $g\left(E_{0}\right)=f$, $g\left(E_{1}\right)=(2 \pi \bar{z})^{-1} W f$.

The general theory guarantees that $g\left(E_{1}\right) \in \delta(C)$ and $g$ is a cochain which is not cohomologous to zero.

The problem of finding the harmonic representative of this class is more difficult.

To produce the corresponding class on the nilmanifold, one merely projects according to the formulae of [5].

\section{BIBLIOGRAPHY}

1. L. Auslander and R. Tolimieri, Abelian harmonic analysis, theta functions and function algebras on a nilmanifold, Lecture Notes in Math., vol. 436, Springer-Verlag, Berlin and New York, 1975.

2. R. Blattner, On induced representations, Amer. J. Math. 83 (1961), 79-98.

3. J. Camora, Représentations du groupe de Heisenberg dans les espaces de $(0, q)$ formes, Math. Ann. 205 (1973), 89-112.

4. P. Cartier, Quantum mechanical commutation relations and theta functions, Algebraic Groups and Discontinuous Subgroups, Proc. Sympos. Pure Math., vol. 9, Amer. Math. Soc., Providence, R. I., 1966, pp. 361-383.

5. L. Corwin, F. Greenleaf and R. C. Penney, A general character formula for irreducible projections on $L^{2}$ of a nilmanifold, Math. Ann. 225 (1977), 21-32.

6. R. Goodman, One parameter groups generated by operators in an enveloping algebra, J. Functional Analysis 6 (1970), 218.

7. H. Grauert, Several complex variables, Springer-Verlag, Berlin and New York, 1976.

8. G. Hochschild and J.-P. Serre, Cohomology of Lie algebras, Ann. of Math. 57 (1953), 591-603.

9. L. Hormander, On the division of distributions by polynomials, Ark. Mat. 3 (1958), 555-568.

10. R. Howe, On Frobenius reciprocity for unipotent algebraic groups over $Q$, Amer. J. Math. 43 (1971), 163-172.

11. On a connection between nilpotent groups and oscillatory integrals associated to singularities, Pacific J. Math. 73 (1977), 329-364.

12. A. Kirillov, Unitary representations of nilpotent Lie groups, Russian Math. Surveys 17 (1962), 53-104.

13. C. Moore and J. Wolf, Square integrable representations of nilpotent groups, Trans. Amer. Math. Soc. 185 (1973), 445-462.

14. H. Moscovici and A. Verona, Harmonically induced representations of nilpotent Lie groups, Invent. Math. 48 (1978), 61-73.

15. H. Moscovici, $A$ vanishing theorem for $L^{2}$-cohomology in the nilpotent case, (Conference on Non-Commutative Harmonic Analysis, Marseille-Luming, June, 1978), Lecture Notes in Math., vol. 728, Springer, Berlin and New York, 1979.

16. E. Nilson, Analytic vectors, Ann. of Math. 70 (1959), 572-615.

17. R. Penney, The structure of ad-associative Lie algebras, Pacific J. Math. (submitted).

18. _ A Fourier transform theorem for nilmanifolds and nil-theta functions, Pacific J. Math. (submitted).

19. N. S. Poulsen, On C-vectors and intertwining bilinear forms for representations of Lie groups, J. Functional Analysis 9 (1972), 87-120.

20. L. Richardson, Decomposition of the $L^{2}$-space of a general compact nilmanifold, Amer. J. Math. 43 (1971), 173-190.

21. I. Satake, Unitary representations of semi-direct product of Lie groups on $\bar{\partial}$-cohomology spaces, Math. Ann. 190 (1971), 177-202.

22. W. Schmid, $L^{2}$ cohomology and the discrete series, Ann. of Math. (2) 103 (1976), 375-394.

Department of Mathematics, Purdue University, West Lafayette, Indiana 47907 